\title{
A MMIC QPSK MODULATOR
}

\author{
PETROS S. TSENES, GIORGOS E. STRATAKOS, S. NIKOLAOS and K. UZUNOGLU \\ Microwave and Fiber Optics Laboratory, Department of Electrical and Computer Engineering, \\ Institute of Communications and Computer Systems, ICCS, \\ National Technical University of Athens, NTUA
}

(Received 6 November 2001; In final form 3 December 2001)

\begin{abstract}
In this paper a MMIC QPSK modulator is described. The bit rate of the baseband signal is $155 \mathrm{Mbits} / \mathrm{sec}$, while the frequency of the carrier is $f_{\mathrm{LO}}=10 \mathrm{GHz}$. The modulation is performed directly at the RF band and therefore an IF stage is not necessary. The design contains all the necessary sub-circuits except for the demultiplexer and the low-pass filters, which are used so that the bandwidth of the binary waves can be reduced. The mixers that are contained in the circuit are not the typical Gilbert cells and as a result they occupy a much smaller area (half). The circuit needs no external LO coupler and no RF chocks. The F-20 process of GEC Marconi was used for designing the modulator. The occupied area is approximately $12 \mathrm{~mm}^{2}$. Section 1 presents fundamentals on Quadriphase Shift Keying (QPSK), while in Section 2 the characteristics of the F-20 process are described. In the following Sections the sub-circuits, the complete circuit, and its simulated results are presented.
\end{abstract}

\section{INTRODUCTION}

\subsection{Band-Pass Data Transmission}

The purpose of a communication system is to transmit information-bearing signals or baseband signals through a communication channel separating the transmitter from the receiver. The term baseband is used to designate the band of frequencies representing the original signal as delivered by a source of information. The efficient utilization of the communication channel requires a shift of the range of baseband frequencies into other frequency ranges suitable for transmission, and a corresponding shift back to the original frequency range after reception. A shift of the range of frequencies in a signal is accomplished by using modulation, which is defined as the process by which some characteristic of a carrier is varied in accordance with a modulating wave. The baseband signal is referred to as the modulating wave and the result of the modulation process is referred to as the modulated wave.

When digital data are required to be transmitted over a band-pass channel, it is necessary to modulate the incoming data onto a carrier wave (usually sinusoidal) with fixed frequency limits imposed by the channel. In any event, the modulation process involves switching or keying the amplitude, frequency or phase of the carrier in accordance with the incoming data. Thus there are three basic signaling techniques known as amplitude-shift keying (ASK), frequency-shift keying (FSK) and phase-shift keying (PSK), which may be viewed

\footnotetext{
* Corresponding author.
} 


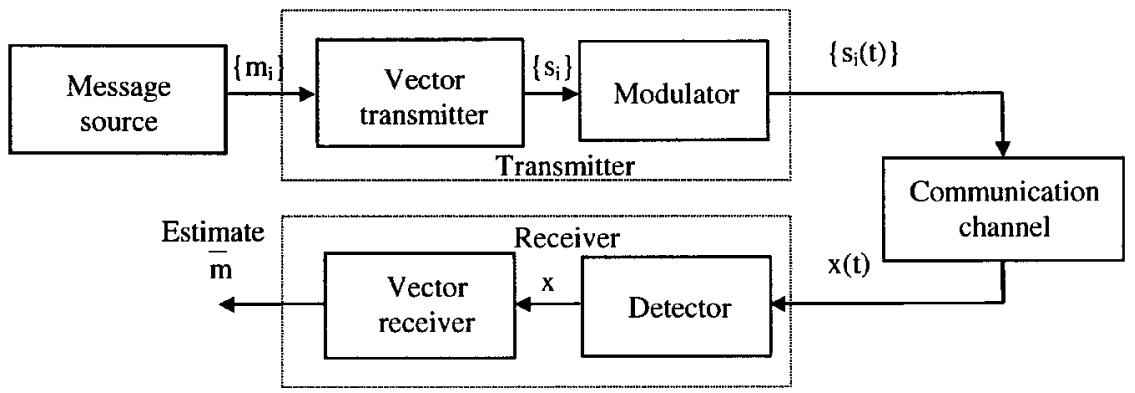

FIGURE 1 Model of band-pass data transmission system.

as special cases of amplitude, frequency and phase modulation, respectively. Ideally. FSK and PSK signals have a constant envelope. This feature makes them impervious to amplitude nonlinearities, as encountered in microwave radio links and satellite channels.

A band-pass data transmission system can be modeled as shown in Figure 1. A message source is assumed to emit one symbol every $T$ seconds, with the symbols belonging to an alphabet of $M$ symbols which we denote by $m_{1}, m_{2}, \ldots, m_{M}$. The a priori probabilities $P\left(m_{1}\right), P\left(m_{2}\right), \ldots P\left(m_{M}\right)$ specify the message source output. If we assume that all $M$ symbols of the alphabet are equally likely, then we may write:

$$
p_{i}=P\left(m_{i}\right)=\frac{1}{M}, \quad \text { for all } i
$$

The output to the message source is presented to a vector transmitter, producing a vector of real numbers. In particular, when the input message $m=m_{i}$, the vector transmitter output takes on the value

$$
s_{i}=\left[\begin{array}{c}
s_{i 1} \\
s_{i 2} \\
\vdots \\
s_{i N}
\end{array}\right], \quad i=1,2, \ldots, M
$$

where $N \leq M$. With this vector as the input the modulator then constructs a distinct signal $s_{i}(t)$ of duration $T$ seconds. The signal is necessarily of finite energy, as shown by

$$
E_{i}=\int_{0}^{T} s_{i}^{2}(t) \mathrm{d} t, \quad i=1,2, \ldots, M
$$

Gram-Schmidt orthogonalization procedure permits the representation of any set of $M$ energy signals as linear combinations of $N$ orthonormal basis functions, where $N \leq M$. So we may represent the given set of real-valued energy signals $s_{1}(t), s_{2}(t), \ldots, s_{M}(t)$, each of duration $T$ seconds, in the form

$$
s_{i}(t)=\sum_{j=1}^{N} s_{i j} \phi_{j}(t), \quad 0 \leq t \leq T \text { and } i=1,2, \ldots, M
$$


where the coefficients of the expansion are defined by

$$
s_{i j}=\int_{0}^{T} s_{i}(t) \phi_{j}(t) \mathrm{d} t, \quad i=1,2, \ldots, M \text { and } j=1,2, \ldots, N
$$

The real-valued basis functions $\varphi_{1}(t), \varphi_{2}(t), \ldots, \varphi_{N}(t)$ are orthonormal.

\subsection{Quadriphase-Shift Keying (QPSK)}

In a coherent binary PSK system, the pair of signals, $s_{1}(t)$ and $s_{2}(t)$, used to represent binary symbols 1 and 0 , respectively, are defined by

$$
\begin{gathered}
s_{1}(t)=\sqrt{\frac{2 E_{b}}{T_{b}}} \cos \left(2 \pi f_{c} t\right) \\
s_{2}(t)=\sqrt{\frac{2 E_{b}}{T_{b}}} \cos \left(2 \pi f_{c} t+\pi\right)=-\sqrt{\frac{2 E_{b}}{T_{b}}} \cos \left(2 \pi f_{c} t\right)
\end{gathered}
$$

where $0 \leq t \leq T_{b}$ and $E_{b}$ is the transmitted signal energy per bit.

As with the binary PSK, quadriphase-shift keying (QPSK) is characterized by the fact that the information carried by the transmitted wave is contained in the phase. In particular, in a QPSK wave the phase of the carrier takes on one of four possible values, such as $\pi / 4,3 \pi / 4,5 \pi / 4$. and $7 \pi / 4$, as shown by

$$
s_{i}(t)=\left\{\begin{array}{cc}
\sqrt{\frac{2 E}{T} \cos \left[2 \pi f_{c} t+(2 i-1) \frac{\pi}{4}\right],} & 0 \leq t \leq T \\
0, & \text { elsewhere }
\end{array}\right.
$$

where $i=1,2,3,4$ and $E$ is the transmitted signal energy per symbol, $T$ is the symbol duration, and the carrier frequency $f_{c}$ equals $n_{c} / T$ for some fixed integer $n_{c}$. Each possible value of the phase corresponds to a unique pair of bits called a dibit. We may rewrite Eq. (8) in the equivalent form

$$
\begin{aligned}
s_{i}(t)= & \sqrt{\frac{2 E}{T}} \cos \left[(2 i-1) \frac{\pi}{4}\right] \cos \left(2 \pi f_{c} t\right)-\sqrt{\frac{2 E}{T}} \sin \left[(2 i-1) \frac{\pi}{4}\right] \sin \left(2 \pi f_{c} t\right), \\
0 & \leq t \leq T \text { and zero elsewhere }
\end{aligned}
$$

where $i=1,2,3,4$. Based on this representation, we may make the following observations:

- There are only two orthonormal basis functions, $\phi_{1}(t)$ and $\phi_{2}(t)$. contained in the expansion of $s_{i}(t)$ and the appropriate form for them is defined by

$$
\phi_{1}(t)=\sqrt{\frac{2}{T}} \cos \left(2 \pi f_{c} t\right) \quad \text { and } \quad \phi_{2}(t)=\sqrt{\frac{2}{T}} \sin \left(2 \pi f_{c} t\right), \quad 0 \leq t \leq T
$$

- There are four message points, and the associated signal vectors are defined by

$$
s_{i}=\left[\begin{array}{c}
\sqrt{E} \cos \left[(2 i-1) \frac{\pi}{4}\right] \\
-\sqrt{E} \sin \left[(2 i-1) \frac{\pi}{4}\right]
\end{array}\right], \quad i=1,2,3,4
$$




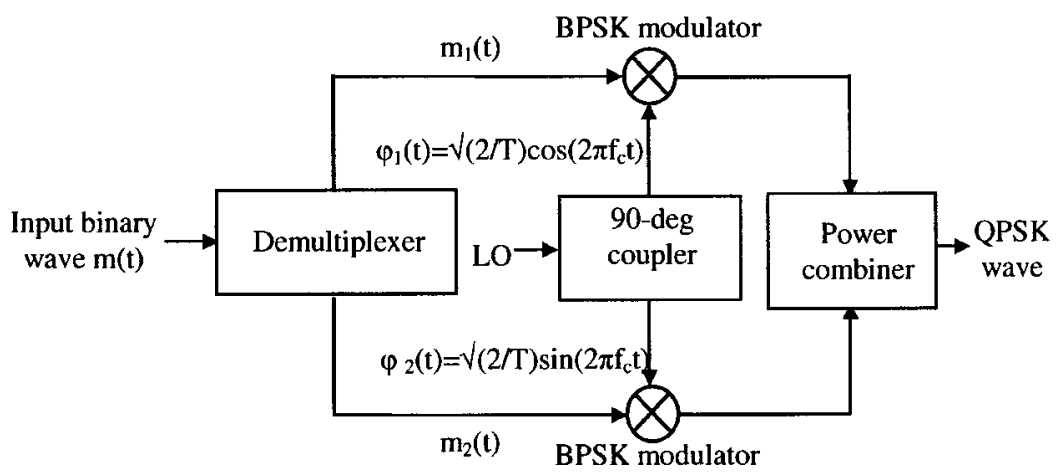

FIGURE 2 Block diagram for a QPSK transmitter.

Figure 2 shows the block diagram of a typical QPSK transmitter. The input binary sequence is represented in polar form, with symbols 1 and 0 represented by $+\sqrt{E_{b}}$ and $-\sqrt{E_{b}}$ volts, respectively. This binary wave is divided by means of a demultiplexer into two separate binary waves consisting of the odd- and even-numbered input bits. These two binary waves are denoted by $m_{1}(t)$ and $m_{2}(t)$. In any signaling interval the amplitudes of $m_{1}(t)$ and $m_{2}(t)$ equal $s_{i 1}$ and $s_{i 2}$, respectively, depending on the particular dibit that is being transmitted. The two binary waves $m_{1}(t)$ and $m_{2}(t)$ are used to modulate a pair of quadrature carriers or orthonormal basis functions $\phi_{1}(t)=\sqrt{2 / T} \cos \left(2 \pi f_{c} t\right)$ and $\phi_{2}(t)=\sqrt{2 / T} \sin \left(2 \pi f_{c} t\right)$. The result is a pair of binary PSK waves, which may be detected independently due to the orthogonality of $\phi_{1}(t)$ and $\phi_{2}(t)$. Finally the two binary PSK waves are added to produce the desired QPSK wave. The symbol duration, $T$, of a QPSK wave is twice as long as the bit duration, $T_{b}$, of the input binary wave. Therefore, for a given bit rate $1 / T_{b}$, a QPSK wave requires half the transmission bandwidth of the corresponding binary PSK wave. Equivalently, for a given transmission bandwidth, a QPSK wave carries twice as many bits of information as the corresponding BPSK wave.

In order to evaluate the spectral properties of a QPSK wave, we assume that the binary wave at the modulator input is ramdom, with symbols 1 and 0 being equally likely, and with the symbols transmitted during adjacent time slots being statistically independent.

Depending on the dibit transmitted during the signaling interval $-T_{b} \leq t \leq T_{b}$, the in-phase component equals $+g(t)$ or $-g(t)$, and similarly for the quadrature component. The $g(t)$ denotes the symbol shaping function, defined by

$$
g(t)=\left\{\begin{array}{cc}
\sqrt{\frac{E}{T}}, & 0 \leq t \leq T \\
0, & \text { elsewhere }
\end{array}\right.
$$

and as a result the in-phase and quadrature components have the same power spectral density, namely, $E \sin ^{2}(T f)[1]$.

The in-phase and quadrature components are statistically independent. Accordingly, the baseband power spectral density of the QPSK signal equals the sum of the individual power spectral densities of the in-phase and quadrature components, and so we may write

$$
S_{B}(f)=2 E \frac{\sin ^{2}(\pi f T)}{(\pi f T)^{2}}=2 E \sin c^{2}(f T)=4 E_{b} \sin c^{2}\left(2 f T_{b}\right)
$$




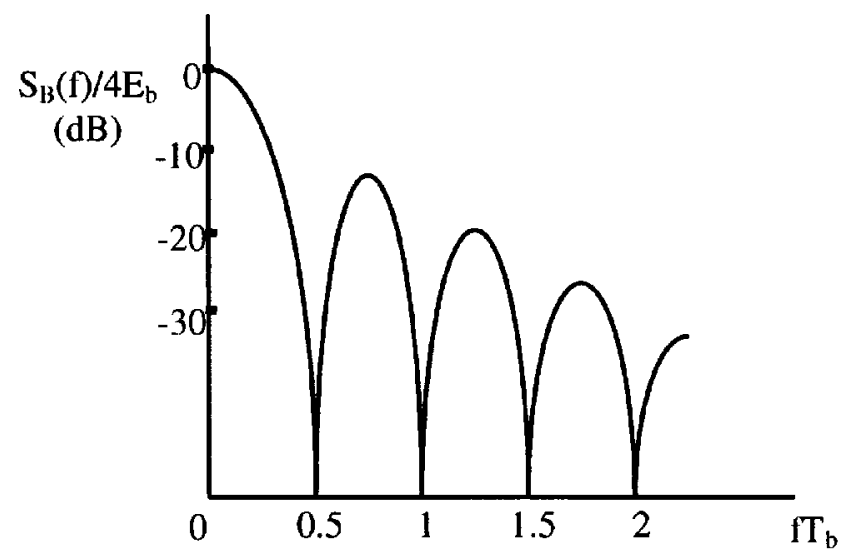

FIGURE 3 Normalized baseband power spectral of QPSK.

Figure 3 shows the baseband power spectral of Eq. (13). The power spectral density is normalized with respect to $4 E_{b}$ and the frequency is normalized with respect to the bit rate $1 / T_{b}$. For $f \gg 1 / T_{b}$, the baseband power spectrum falls off as the inverse square of frequency.

In our application the frequency of the carrier is $f_{c}=10 \mathrm{GHz}$, while the rate of the baseband signal is $155 \mathrm{Mbits} / \mathrm{sec}$ which means that $T_{b}=1 / 155 \mu \mathrm{sec}$. Section 3 describes the power combiner which adds the two binary PSK waves. In Section 4 the 90-deg coupler which is necessary for the production of the two orthonormal basis functions is described. In Section 5 the BPSK modulator will be described and finally in Section 6 the simulated results of the QPSK modulator will be given. The QPSK modulator that was designed contains no demultiplexer and no filters. It is obvious that the passband of the lowpass filters that will externally be used, will determine the actually useful bandwidth of the QPSK wave.

\section{F-20 PROCESS OF GEC MARCONI}

The materials' technology foundry of GEC Marconi has developed an advanced GaAs technology, which is used exclusively for designing Monolithic Microwave Integrated Circuits (MMICs) and affords a remarkable repeatability and reliability. The F-20 process provides a complete smart library, which contains linear and nonlinear models of MESFETs and models of different structures of capacitors, inductors and resistors.

The gate length of the MESFETs of F-20 process is $0.5 \mu \mathrm{m}$ and its cut-off frequency is $20 \mathrm{GHz}$ [2]. The linear models of the MESFETs are based on measurements and are valid only for certain dc bias points and certain gate widths. As a matter of fact they are tables of the $s$-parameters of the MESFETs $v s$. frequency. The gate of a MESFET is divided into multiple fingers, while each finger has the same length. There are several models for different numbers of fingers and lengths. These models provide a linear output power $v s$. input power, independent of the value of the input power. Therefore, the compression point cannot be defined. The nonlinear models should be used if the compression point should be defined or if the extrapolation of the I-V curves is necessary. It is obvious that in our case (design of a modulator) the nonlinear models were used.

The F-20 process supports two kinds of capacitors: nitride capacitors and polyimide capacitors. Their form is quadrangular, while their dimensions should be between $50 \mu \mathrm{m} \times 50 \mu \mathrm{m}$ 
and $350 \mu \mathrm{m} \times 350 \mu \mathrm{m}$. The range of values for the polyimide capacitors is between $0.06 \mathrm{pF}$ and $2.5 \mathrm{pF}$, while for the nitride capacitors is between $0.5 \mathrm{pF}$ and $59 \mathrm{pF}$.

The rectangular spiral inductors are fabricated on the second metal layer. There are different kinds of inductors, while each of them has a different line spacing. An inductor should not have more than 7 and less than 1 spirals, and the range of values for the spiral inductors is between $1 \mathrm{nH}$ and $13 \mathrm{nH}$.

There are two kinds of resistors: the mesa resistors and the nichrome resistors. Given that the resistivity of the two materials is $300 \Omega$ /sq and $50 \Omega /$ sq, respectively, it can be estimated that the values of the mesa resistors are between $10 \Omega$ and $10 \mathrm{k} \Omega$, while the values of the nichrome resistors are between $5 \Omega$ and $2 \mathrm{k} \Omega$.

Finally transmission lines can be fabricated on the first metal layer as well as on the second metal layer. The second metal layer is usually used because it has significantly less resistivity than the first layer. Moreover, the smart library contains a number of elements like T-junctions and cross-junctions, vias and DC- and RF-pads.

\section{WILKINSON COMBINER (POWER COMBINER)}

As it has already been seen (Fig. 2) the two binary PSK waves are added, by a power combiner, to produce the desired QPSK wave. A power combiner has exactly the same form as a power divider. The only difference between them is that the outputs of a divider are actually the inputs of a combiner and vice versa. A power combiner must be a three port device having a special set of characteristics: (1) all ports are matched, (2) RF power applied to the input ports is added (without phase difference) and extracted from the output port, (3) input ports are isolated. Therefore a power combiner-divider has the S matrix:

$$
S=-\frac{1}{\sqrt{2}}\left[\begin{array}{lll}
0 & 1 & 1 \\
1 & 0 & 0 \\
1 & 0 & 0
\end{array}\right]
$$

The most common form of a power combiner is the Wilkinson combiner (Fig. 4). As it can be seen it consists of three transmission line sections, which are 0.25 wavelengths, and a resistor which value is $R=2 \cdot Z_{0}$, where $Z_{0}$, is the port impedance (usually $50 \Omega$ ). The characteristic impedance of the symmetrical transmission line sections is $Z_{\mathrm{o}}^{\prime}=\sqrt{2} Z_{\mathrm{o}}$, while the characteristic impedance of the third transmission line section is $Z_{0}$. The presence of the third transmission line section is not necessary and if it is not used then the value of the $s_{11}$-parameter of the combiner is somewhat reduced, but still very satisfying. Therefore, in order to reduce the occupied area, we did not use the third transmission line section.

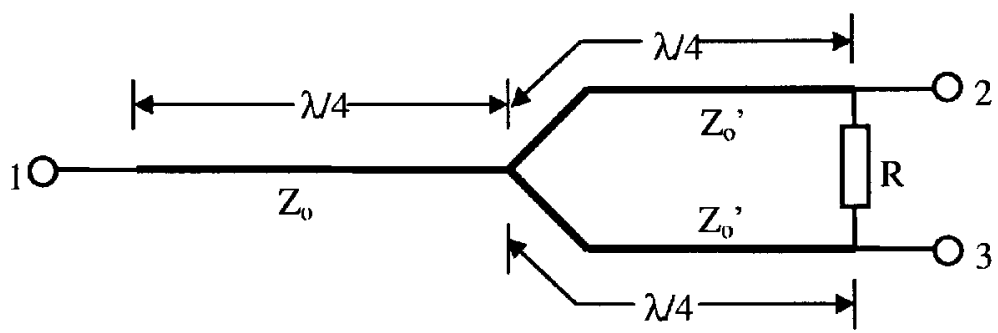

FIGURE 4 Wilkinson combiner/divider. 
It can easily be calculated that the length of the transmission line sections, which are required in our application, is $(\lambda / 4)=2500 \mu \mathrm{m}$. The usage of shorter transmission line sections would be very convenient. It is known that a distributed inductance connected with a distributed shunt capacitance is a very good model of a transmission line. Therefore a shorter transmission line would have smaller inductance and capacitance. The reduction of the inductance can easily be balanced using increased characteristic impedance, while the reduction of the capacitance can be balanced using two lumped, shunt capacitances at either end (Fig. 5).

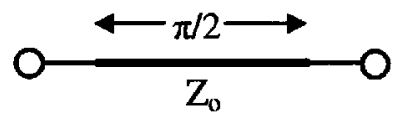

(a)

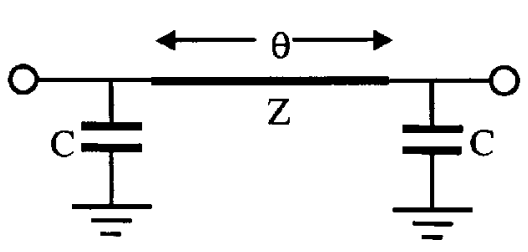

(b)

FIGURE 5 (a) $\lambda / 4$ wavelength transmission line, (b) equivalent topology using a transmission line of electrical length $\theta<\pi / 2$ and characteristic impedance $Z>Z_{\mathrm{o}}$ and two shunt capacitors $C$.

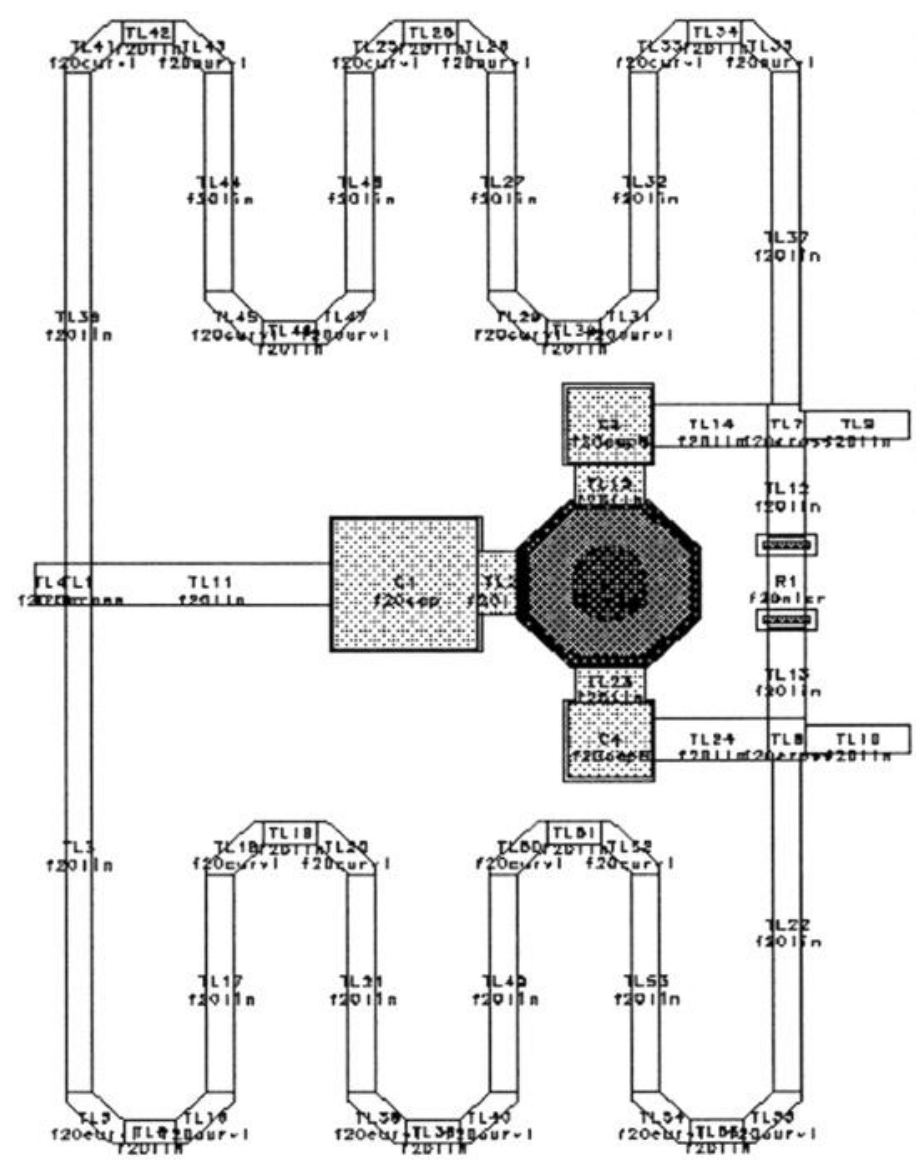

FIGURE 6 Final layout of the Wilkinson combiner. 


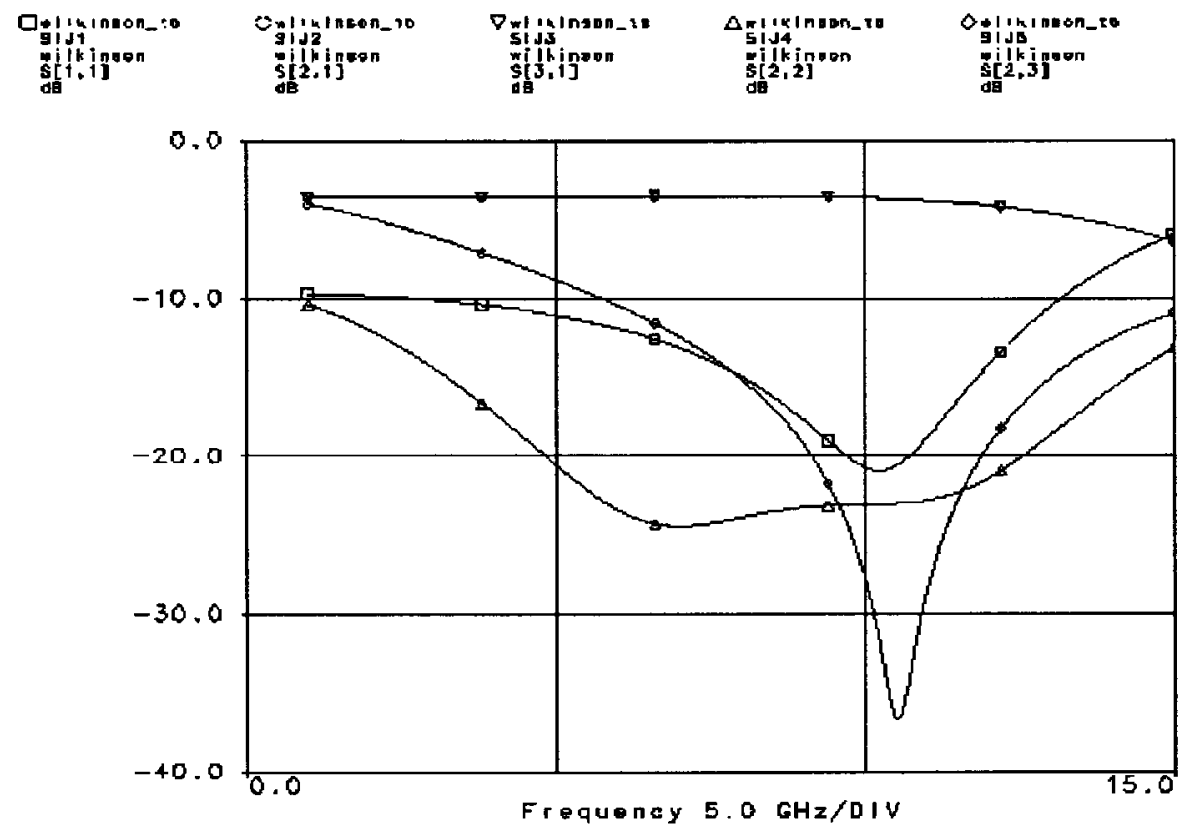

FIGURE 7 Wilkinson combiner's s-parameters $v s$. frequency.

The relations between $Z, Z_{\mathrm{o}}, C$ and $\theta$ can easily be determined [3]:

$$
Z=\frac{Z_{\mathrm{o}}}{\sin \theta} \quad \text { and } \quad \omega C=\frac{\cos \theta}{Z_{\mathrm{o}}}
$$

Equation (15) show that electrical length can be smaller if the values of the characteristic impedance $Z$ and the capacitors $C$ are increased. This topology is very convenient for MMIC designs because the required lumped capacitors can easily be realized. This technique was used so as the Wilkinson combiner to occupy a much smaller area. The quarter-wavelength transmission sections were replaced by the equivalent circuit of Figure 5(b).

Figure 6 shows the Wilkinson combiner's final layout. It occupies the smallest possible area and at the same time it is placed in such a way that can be easily connected with the other sub-circuits of the design.

In Figure 7 the transmission coefficients $\left(\left|s_{21}\right|\right.$ and $\left.\left|s_{31}\right|\right)$, the isolation $\left(\left|s_{23}\right|\right)$ between the input ports and the reflection coefficients $\left(\left|s_{11}\right|\right.$ and $\left.\left|s_{22}\right| \equiv\left|s_{33}\right|\right)$ can be seen. In Table I the exact values of the $s$-parameters of the combiner into the desired frequency range are presented.

TABLE I Exact Values of the $s$-parameters in the Desired Frequency Range.

\begin{tabular}{lllll}
\hline & \multicolumn{4}{c}{ s-parameters $(\mathrm{dB})$} \\
\cline { 2 - 5 } Frequency $(\mathrm{GHz})$ & $\left|s_{11}\right|$ & $\left|s_{22}\right|=\left|s_{33}\right|$ & $\left|s_{23}\right|$ & $\left|s_{21}\right|=\left|s_{31}\right|$ \\
\hline 9.9 & -44.42 & -30.68 & -32.90 & -3.11 \\
10.0 & -38.68 & -30.62 & -35.21 & -3.11 \\
10.1 & -35.25 & -30.56 & -38.32 & -3.11 \\
\hline
\end{tabular}




\section{90-DEG COUPLER}

As it has already been noticed (Fig. 2) the two orthonormal basis functions $\phi_{1}(t)=\sqrt{2 / T} \cos \left(2 \pi f_{c} t\right)$ and $\phi_{2}(t)=\sqrt{2 / T} \sin \left(2 \pi f_{c} t\right)$ are produced by a 90 -deg hybrid. A microwave 90 -deg hybrid coupler is a four port device having a special set of characteristics: (1) all ports are matched, (2) RF power applied to any one port is split equally between two of the other ports, (3) the signals at the output ports have an 90-deg phase difference and (4) the remaining port is isolated. Therefore an ideal $90-\mathrm{deg}$ hybrid has the $\mathrm{S}$ matrix

$$
\mathrm{S}_{90}=\frac{1}{\sqrt{2}}\left[\begin{array}{cccc}
0 & 0 & -\mathrm{j} & 1 \\
0 & 0 & 1 & -\mathrm{j} \\
-\mathrm{j} & 1 & 0 & 0 \\
1 & -\mathrm{j} & 0 & 0
\end{array}\right]
$$

Equation (16) implies specific phase shifts between the input and output ports. In real hybrids, the input-to-output phase shift is rarely important, but the phase difference between the two output ports is critical. Real hybrids differ from the ideal hybrid described above in several ways: the most important nonidealities are phase and amplitude balance, loss and VSWR. Balance refers to the matching of phase and power levels at any two output ports. Phase balance is the deviation in phase from the ideal phase difference between any pair of outputs, and amplitude balance is the difference in output amplitude, usually expressed in $\mathrm{dB}$. Isolation is the ratio of power at the isolated port to that applied at the input. Isolation and balance are usually frequency-dependent and may be different for different pairs of ports. They are generally the same only if the hybrid has a symmetrical structure. Like all other real components, microwave hybrids introduce some dissipative power loss, which is usually specified as loss above the unavoidable $3 \mathrm{~dB}$ of coupling loss.

One of the simplest 90-deg coupler to design is the branch-line hybrid (Fig. 8). where coupling is achieved between two transmission lines by periodic interconnecting branches [4]. The interconnecting lines are one-quarter wavelength long and are spaced one-quarter wavelength apart. The characteristic impedance of the series lines between the branches is $\sqrt{2} Z_{\mathrm{o}}$ and the branch impedance is simply $Z_{\mathrm{o}}$ the terminating impedance of the four ports.

The technique that was discussed in the previous section was used so as the 90-deg coupler to occupy as small area as possible and the results were quite good. In Table II the exact values of all important $s$-parameters of the coupler at the desired frequency $\left(f_{c}=10 \mathrm{GHz}\right)$ are presented. Moreover the exact value of the phase difference between the two output ports at the desired frequency is $89.8^{\circ}$.

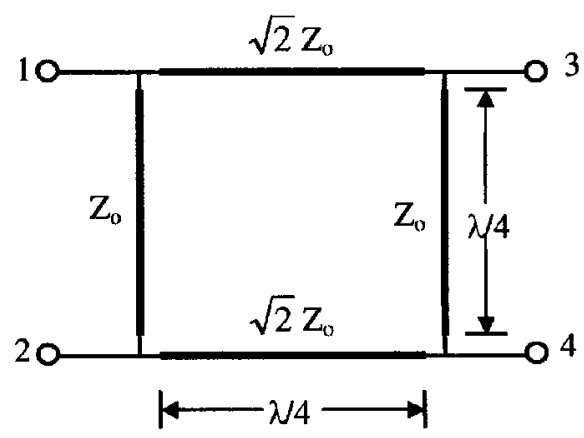

FIGURE 8 Branch-line hybrid. 
TABLE II Exact Values of All Important s-parameters of the Branch-Line Hybrid at the Desired Frequency.

\begin{tabular}{llllllll}
\hline \multicolumn{7}{c}{ s-parameters $(d B)$} \\
\hline$\left|s_{11}\right|$ & $\left|s_{22}\right|$ & $\left|s_{33}\right|$ & $\left|s_{44}\right|$ & $\left|s_{21}\right|$ & $\left|s_{31}\right|$ & $\left|s_{41}\right|$ & $\left|s_{34}\right|$ \\
\hline-20.30 & -22.28 & -21.35 & -18.67 & -20.01 & -4.31 & -4.48 & -20.22 \\
\hline
\end{tabular}

It is known that the basic principle of a one-quarter wavelength long line is that there is a 90-deg phase difference between the input and the output wave. Therefore a Wilkinson divider and an additional $\lambda / 4$ long transmission line could be used so as the two orthonormal functions to be produced. This form is not very popular because it is very narrowband and introduces amplitude balance. Being narrowband is not a problem in our application because the 90-deg coupler should have an appropriate performance only at the LO frequency $\left(f_{c}=10 \mathrm{GHz}\right)$. The amplitude balance can be eliminated using an unbalanced Wilkinson divider instead of a balanced one. That means that the branches of the divider do not have the same characteristic impedance or in other words the same line width. Using at the same time the same technique that was used for the branch-line hybrid, the occupied area is now much smaller. Figure 9

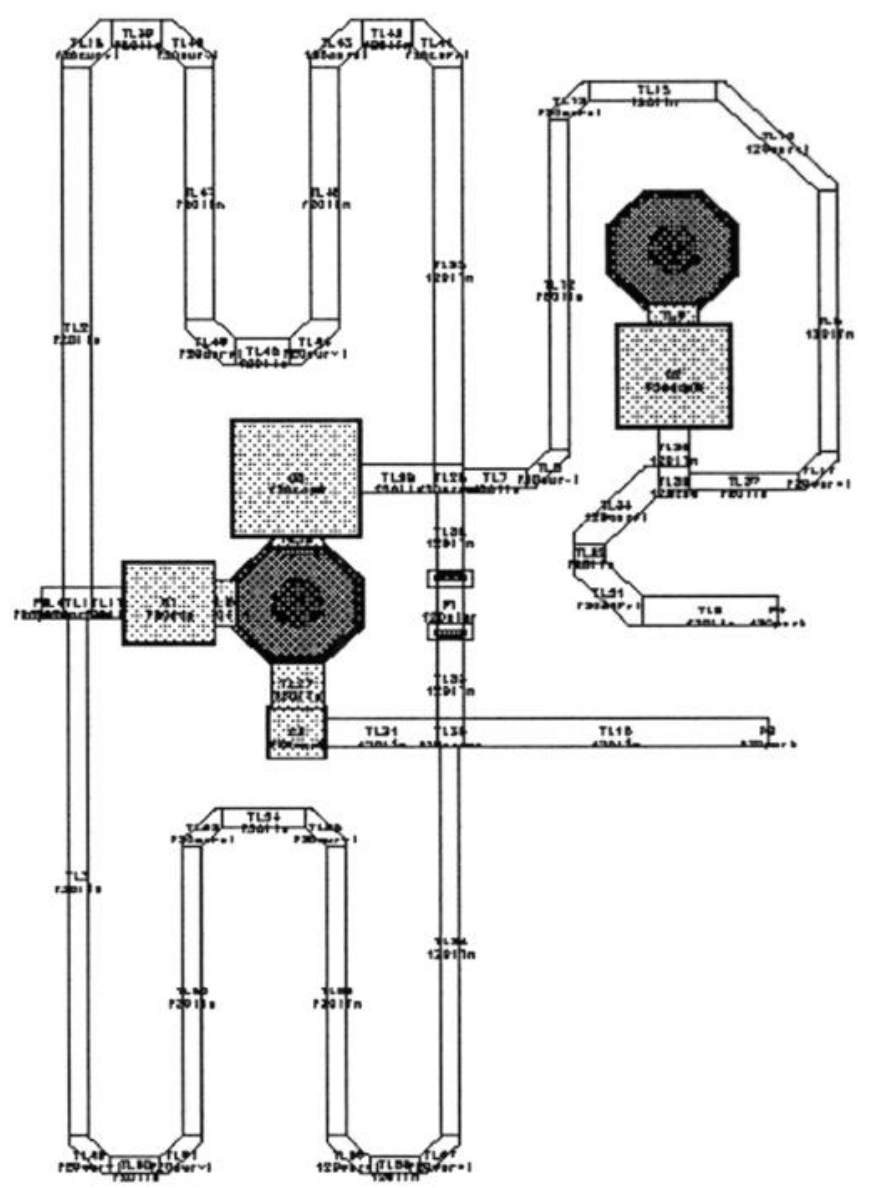

FIGURE 9 Final layout of the 90-deg coupler using a Wilkinson divider and a $\lambda / 4$ long transmission line. 


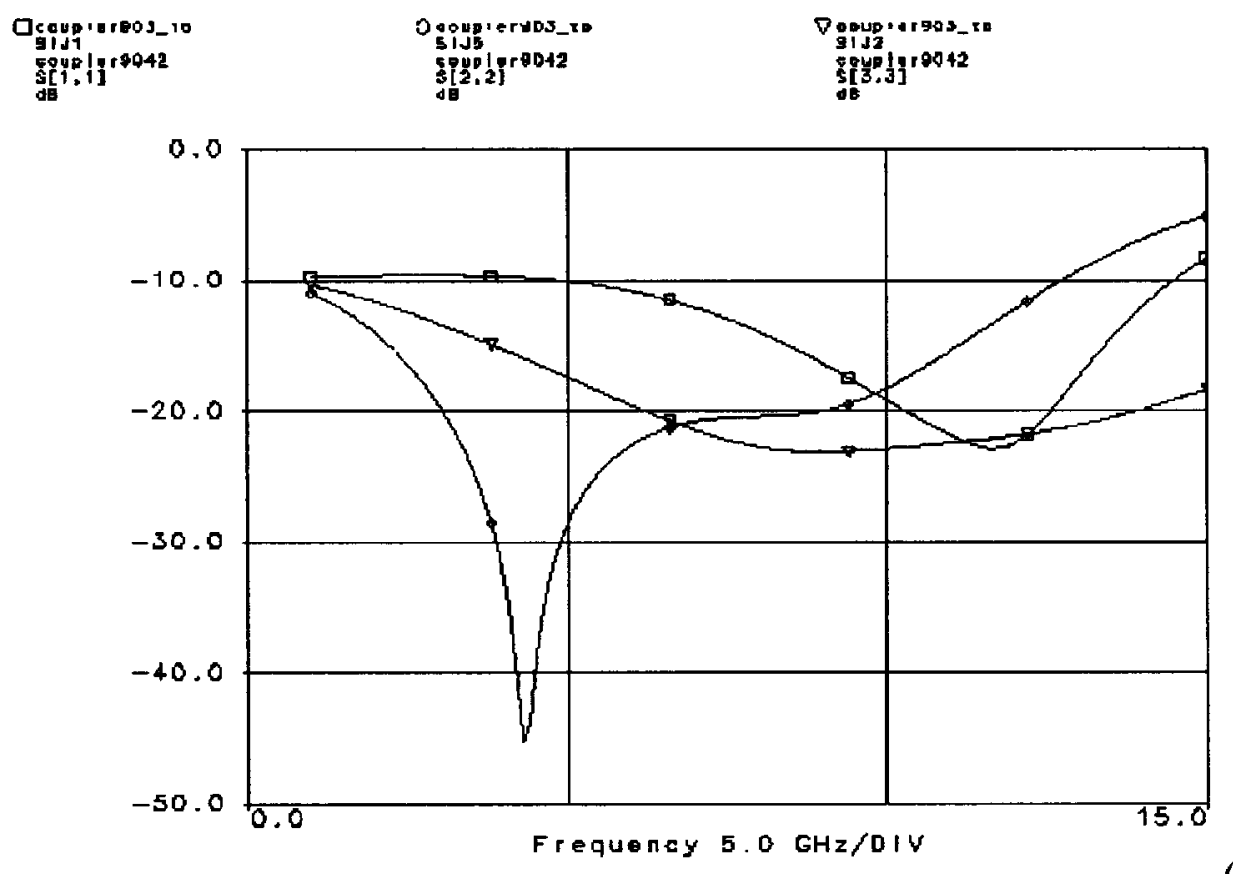

(a)
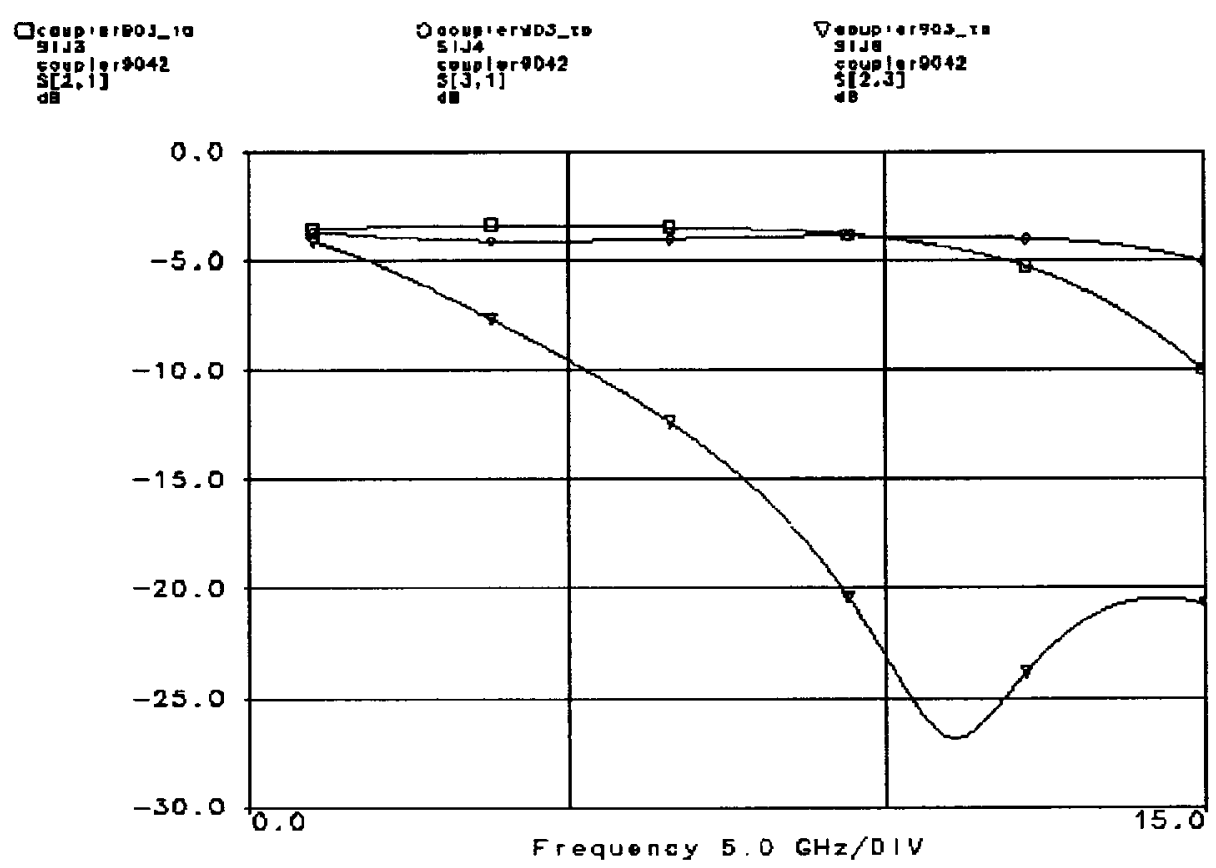

(b)

FIGURE 10 (a) $\left|s_{11}\right|,\left|s_{22}\right|$ and $\left|s_{33}\right|$ parameters of the 90-deg coupler. (b) $\left|s_{21}\right|,\left|s_{31}\right|$ and $\left|s_{23}\right|$ parameters of the 90-deg coupler. 
TABLE III Exact Values of All s-parameters of the Final 90-deg Coupler Design at the Desired Frequency.

\begin{tabular}{lccccc}
\hline \multicolumn{6}{c}{ s-parameters $(d B)$} \\
\hline$\left|s_{11}\right|$ & $\left|s_{22}\right|$ & $\left|s_{33}\right|$ & $\left|s_{21}\right|$ & $\left|s_{31}\right|$ & $\left|s_{23}\right|$ \\
\hline-19.21 & -18.29 & -22.93 & -3.96 & -3.87 & -23.14 \\
\hline
\end{tabular}

shows the 90-deg coupler's final layout. It occupies the smallest possible area and at the same time it is placed in such a way that can be easily connected with the other sub-circuits of the design.

In Figure 10(a) the reflection coefficients $\left(\left|s_{11}\right|,\left|s_{22}\right|\right.$ and $\left.\left|s_{33}\right|\right)$ can be seen, while in Figure $10(\mathrm{~b})$ the transmission coefficients $\left(\left|s_{21}\right|\right.$ and $\left.\left|s_{31}\right|\right)$ and the isolation $\left(\left|s_{23}\right|\right)$ are presented.

Moreover the exact value of the phase difference between the two output ports at the desired frequency is $90^{\circ}$. In Table III the exact values of all $s$-parameters of the coupler at the desired frequency $\left(f_{c}=10 \mathrm{GHz}\right)$ are presented.

Comparing Tables II and III it is obvious that the 90-deg coupler that uses a Wilkinson divider has better performance than the branch-line hybrid. In addition to the smaller occupied area, the dissipative power loss (above the unavoidable $3 \mathrm{~dB}$ of coupling loss) is much smaller.

\section{BPSK-MODULATOR}

\subsection{Introduction}

The BPSK modulators are necessary for the production of the binary PSK waves, which then are added so as the QPSK wave to be produced. A BPSK modulator is actually a balanced mixer and particularly in our design a single balanced mixer, because it occupies smaller substrate area than a double balanced one. It consists of three MESFETs and a 180-deg coupler (Fig. 11). The baseband signal and its reverse $\left(m_{i}(t)\right.$ and $\left.-m_{i}(t)\right)$ are applied to the gates of $\mathrm{Q}_{1}$ and $\mathrm{Q}_{2}$ MESFETs which operate as switches, while the LO signal is applied to the gate of $\mathrm{Q}_{3}$ MESFET which operate as a current source.

Figure 11(a) shows the operation of the BPSK modulator at the time slot, when the baseband signal is logic ' 1 ' and its amplitude is $+\mathrm{A}$. The LO signal $\left(\varphi_{i}(t)\right)$ goes "through" the $\mathrm{Q}_{1}$ MESFET, is applied to the $0^{\circ}$-port of the 180-deg coupler and is extracted from the coupler having a phase difference $\vartheta^{\circ}$. Figure 11(b) shows the operation of the BPSK modulator at the time slot, when the baseband signal is logic ' 0 ' and its amplitude is $-\mathrm{A}$. The LO signal $\left(\varphi_{i}(t)\right)$ goes "through" the $\mathrm{Q}_{2}$ MESFET, is applied to the $180^{\circ}$-port of the 180 deg coupler and is extracted from the coupler having a phase difference $180^{\circ}+\vartheta^{\circ}$. So, it is obvious that this circuit operates as a BPSK modulator. Its output signal is a sinusoid with constant amplitude and its phase has two possible values corresponding to binary symbols ' 1 ' and ' 0 '.

\subsection{0-Deg Coupler}

A microwave 180-deg hybrid coupler is used for the combination of the drains of $\mathrm{Q}_{1}$ and $\mathrm{Q}_{2}$ MESFETs. It is a four port device having a special set of characteristics: (1) all ports are matched, (2) RF power applied to any one port is split equally between two of the other 

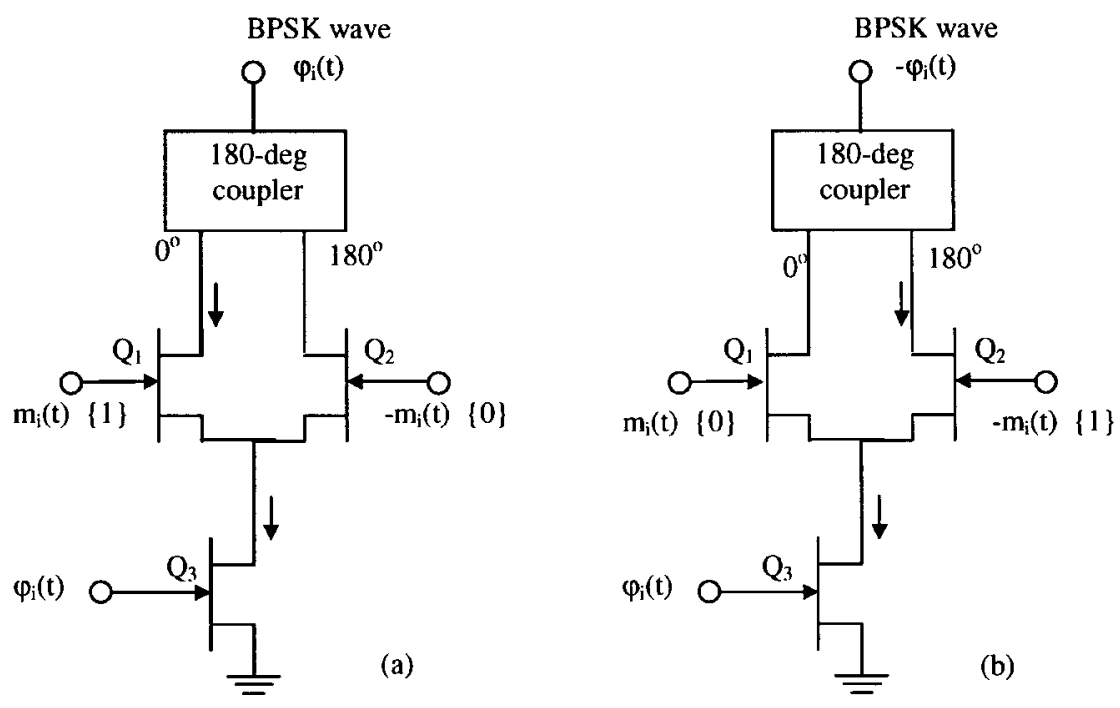

FIGURE 11 Operation of the BPSK modulator when the input signal represents (a) logic ' 1 ', (b) logic ' 0 '.

ports, (3) the signals at the output ports have an 180-deg phase difference and (4) the remaining port is isolated.

The properties of the 180-deg coupler are illustrated in Figure 12. The lines between the ports show the phase shift between them. For example, if a signal is applied to port 4 of the ideal 180-deg hybrid, it appears at ports 2 and 3 with identical phases and at a level $3 \mathrm{~dB}$ below the input. No output appears at port 1 . Similarly, if port 1 is excited, the outputs are ports 2 and 3, and port 4 has no output. The outputs are $180 \mathrm{deg}$ out of phase, and, of course, are $3 \mathrm{~dB}$ lower in level than the input. In this hybrid, ports 3 and 2 are often called the sum (sigma) and difference (delta) ports, because the output voltage at port 3 is proportional to the sum of the input voltages at ports 1 and 4 , and the voltage at port 2 is proportional to their difference. Similarly, ports 4 and 1 can be considered the sum and

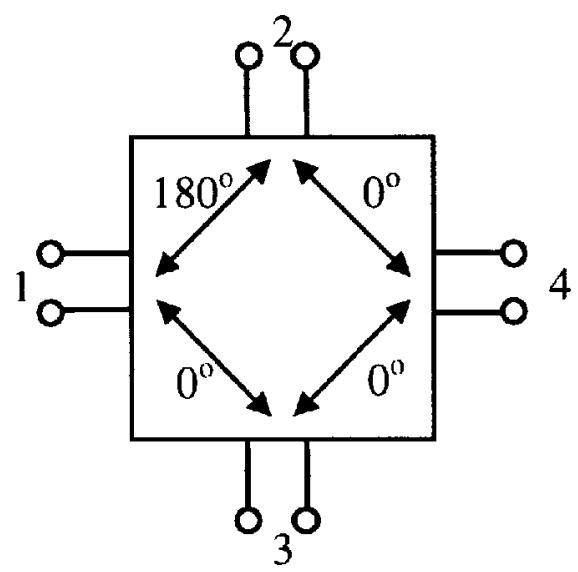

FIGURE 12 Ideal 180-deg hybrid. 
difference ports, respectively, with ports 2 and 3 the inputs. Therefore an ideal 180-deg hybrid has the S matrix

$$
\mathrm{S}_{180}=\frac{1}{\sqrt{2}}\left[\begin{array}{cccc}
0 & -1 & 1 & 0 \\
-1 & 0 & 0 & 1 \\
1 & 0 & 0 & 1 \\
0 & 1 & 1 & 0
\end{array}\right]
$$

Equation (17) implies specific phase shifts between the input and output ports. In real hybrids, the input-to-output phase shift is rarely important, but the phase difference between the two output ports is critical.

One of the simplest 180-deg coupler to design is the so-called "rat-race" or ring hybrid (Fig. 13). It consists of four transmission line parts; three of them are 0.25 wavelengths while the fourth is 0.75 wavelengths. The characteristic impedance of these parts is $\sqrt{2} Z_{0}$ where $Z_{\mathrm{o}}$ is the port impedance (usually $50 \Omega$ ).

The technique that was discussed in Section 3, where the quarter-wavelength transmission sections are replaced by the equivalent circuit of Figure 5(b), is used so as the 180-deg coupler to occupy as small area as possible. Figure 14 shows the 180-deg coupler's final layout. It occupies the smallest possible area and at the same time it is placed in such a way that can easily be connected with the other sub-circuits of the design.

In Figure 15 the transmission coefficients $\left(\left|s_{21}\right|,\left|s_{31}\right|\right.$ and $\left.\left|s_{41}\right|\right)$ and the isolation $\left(\left|s_{23}\right|\right)$ between the output ports can be seen. In Table IV the exact values of all important $s$-parameters of the coupler into the desired frequency range are presented.

The phase difference between the two output ports at the central frequency is almost $180.6^{\circ}$, while the total deviation of its value for a $200 \mathrm{MHz}$ frequency range around the central frequency is smaller than $2^{\circ}$.

We also designed the 180-deg coupler using the technique that we finally used for designing the 90-deg coupler. That means that an unbalanced Wilkinson combiner and an one-half wavelength long line were used. The performance of this design was quite satisfying except for the phase difference. The total deviation of its value for a $200 \mathrm{MHz}$ frequency range around the central frequency was greater than $4.5^{\circ}$, which was unacceptable and that is why this design was not finally used.

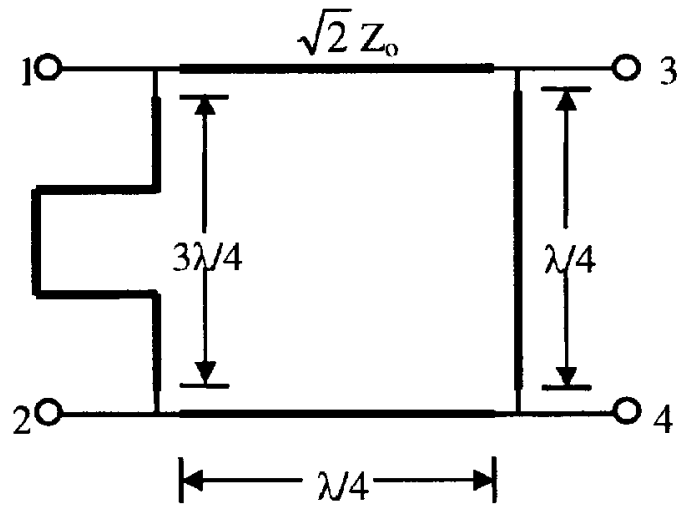

FIGURE 13 A microwave 180-deg hybrid coupler. 


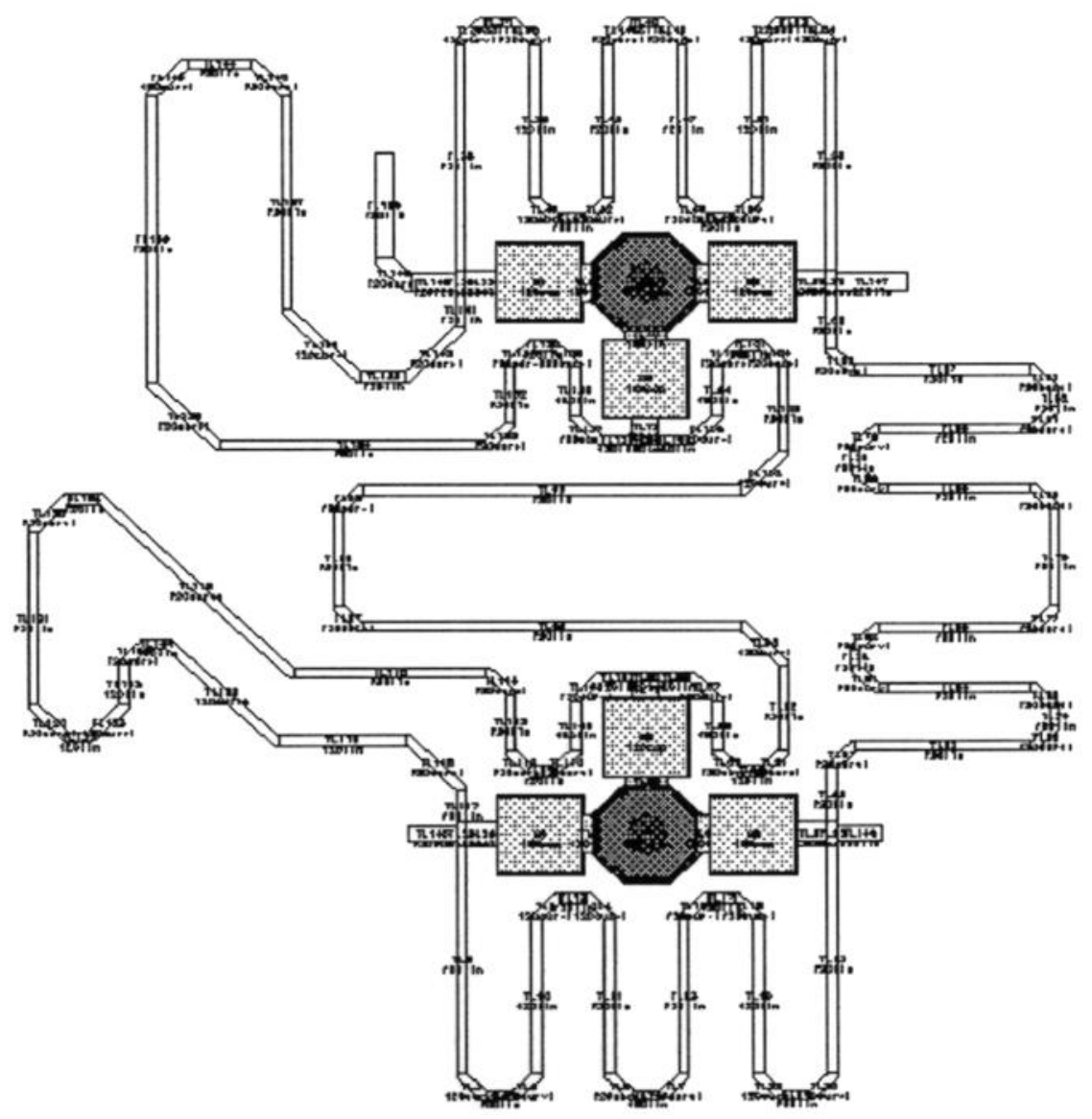

FIGURE 14 Final layout of the 180-deg coupler.

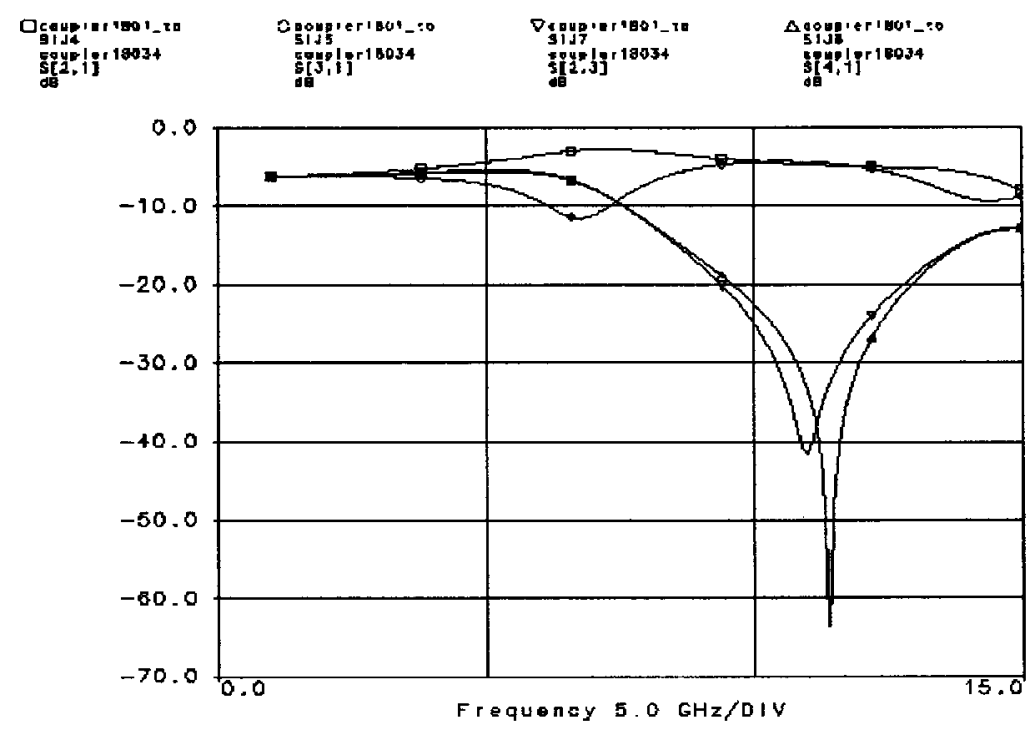

FIGURE 15 Transmission coefficients $\left(\left|s_{21}\right|,\left|s_{31}\right|\right.$ and $\left.\left|s_{41}\right|\right)$ and isolation $\left(\left|s_{23}\right|\right)$ between the output ports. 
TABLE IV Exact Values of All s-parameters of the Final 180-deg Coupler Design at the Desired Frequency.

\begin{tabular}{lcccccccc}
\hline & \multicolumn{7}{c}{ s-parameters $(\mathrm{dB})$} \\
\cline { 2 - 8 } Frequency $(\mathrm{GHz})$ & $\left|s_{11}\right|$ & $\left|s_{22}\right|$ & $\left|s_{33}\right|$ & $\left|s_{44}\right|$ & $\left|s_{41}\right|$ & $\left|s_{23}\right|$ & $\left|s_{21}\right|$ & $\left|s_{31}\right|$ \\
\hline 9.9 & -24.2 & -18.8 & -26.3 & -23.9 & -21.8 & -24.0 & -4.23 & -4.43 \\
10.0 & -24.9 & -19.1 & -27.0 & -24.4 & -22.5 & -25.0 & -4.28 & -4.39 \\
10.1 & -25.8 & -19.5 & -27.6 & -24.9 & -23.2 & -26.0 & -4.34 & -4.37 \\
\hline
\end{tabular}

\subsection{Active Part of the BPSK Modulator}

For the designing of the active part of the BPSK modulator the nonlinear model of the $4 \times 75$ MESFET was used. As it has already been mentioned $\mathrm{Q}_{3}$ MESFET is the current source while $\mathrm{Q}_{1}$ and $\mathrm{Q}_{2}$ operate as switches (Fig. 16). Therefore the latter MESFETs must be biased near the cut-off region, while $\mathrm{Q}_{3}$ MESFET must be biased at a point where its dc current is double of the dc current of the other two MESFETs. It was chosen $V_{\mathrm{DS} 1}=V_{\mathrm{DS} 2}=2.5 \mathrm{~V}$ and $V_{\mathrm{GS} 1}=V_{\mathrm{GS} 2}=-1.1 \mathrm{~V}$ and therefore the dc current is $I_{\mathrm{D} 1}=I_{\mathrm{D} 2}=5.2 \mathrm{~mA}$. As a result the required amplitude of the binary waveform is only $0.4 \mathrm{~V}$. Moreover the dc bias point of $\mathrm{Q}_{3}$ MESFET has $V_{\mathrm{DS} 3}=2.5 \mathrm{~V}$ and $V_{\mathrm{GS} 3}=-0.9 \mathrm{~V}$ so as the dc current to be $\mathrm{I}_{\mathrm{D} 3}=2.1_{\mathrm{D} 1,2}=10.4 \mathrm{~mA}$.

Given that $V_{\mathrm{DS} 3}=2.5 \mathrm{~V}$ and $V_{\mathrm{GS} 1,2}=-1.1 \mathrm{~V}$ it can easily be calculated that $V_{\mathrm{G} 1,2}=V_{\mathrm{DS} 3}+V_{\mathrm{GS} 1,2}=1.4 \mathrm{~V}$. Moreover $V_{\mathrm{D} 1,2}=V_{\mathrm{DS} 1,2}+V_{\mathrm{DS} 3}=5 \mathrm{~V}$ which means that the required dc external voltages are $V_{\mathrm{D} 1,2}=5 \mathrm{~V}, V_{\mathrm{G} 1,2}=1.4 \mathrm{~V}$ and $V_{\mathrm{G} 3}=-0.9 \mathrm{~V}$. Considering that only $V_{1}=+5 \mathrm{~V}$ and $V_{2}=-5 \mathrm{~V}$ are the available external dc voltages, the required dc voltages can be produced by voltage dividers. In Figure 16, in addition to the voltage dividers, two quarter-wavelength long lines can be seen. They prevent RF signal from

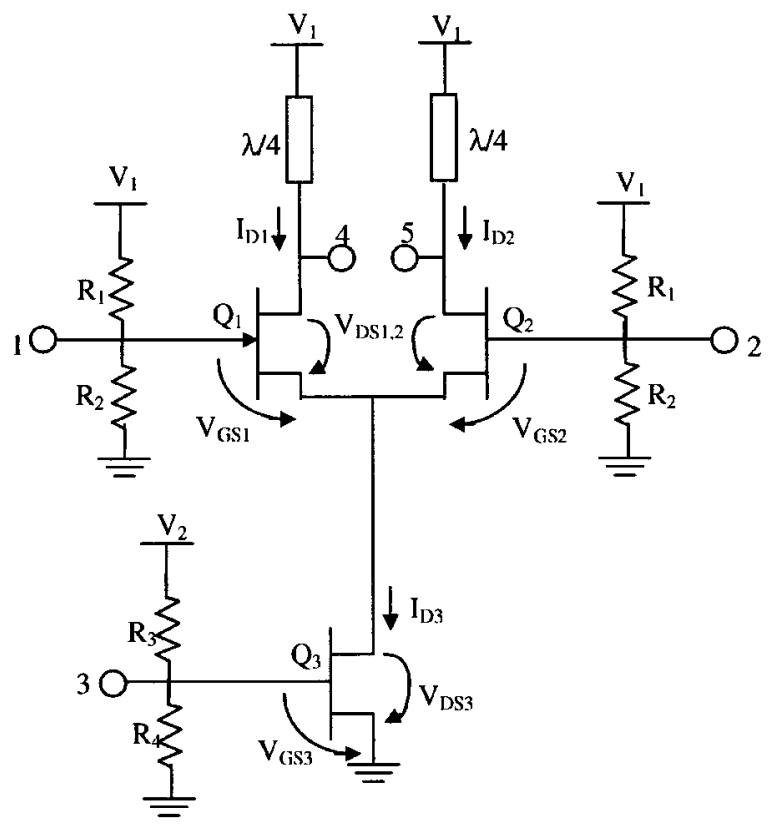

FIGURE 16 DC biased active part of the BPSK modulator. 


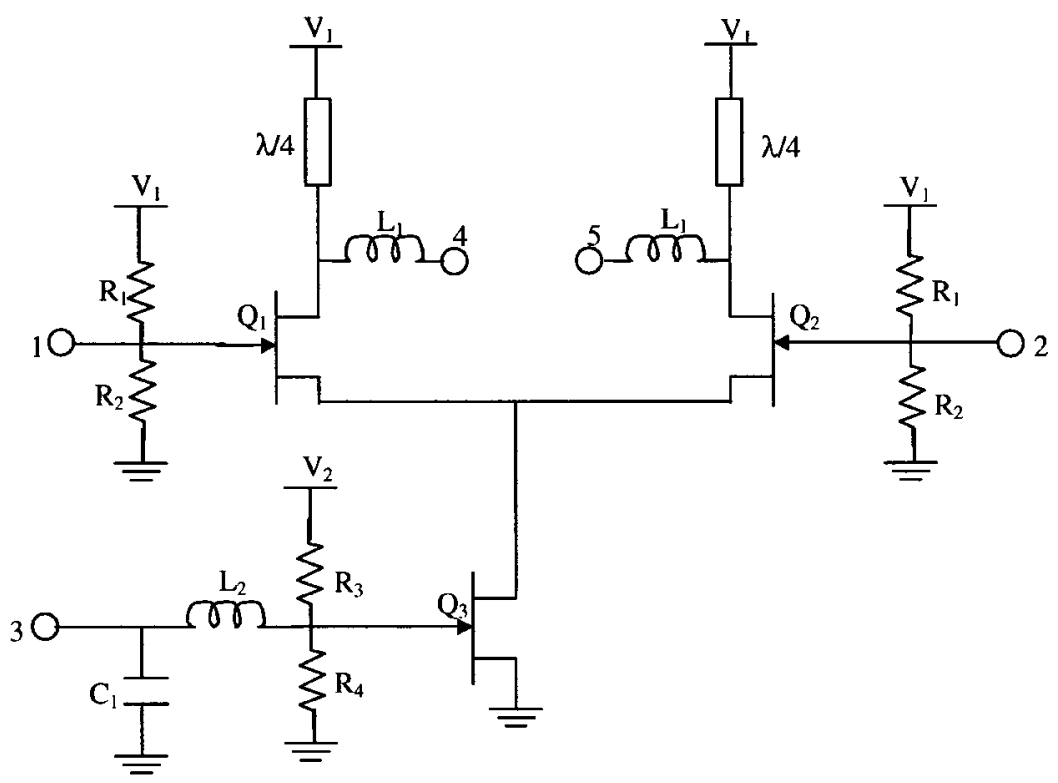

FIGURE 17 DC biased and matched active part of the BPSK modulator.

going into the dc power supply that would mean that the BPSK modulator could have no RF output signal.

The circuit that is shown in Figure 16 does not have matched ports. It is obvious that the optimum performance of the circuit requires matched ports (Fig. 17). To minimize the occupied area of the substrate, the simplest sub-circuits were used in the design.

Table V shows the values of the reflection coefficients at LO and output ports in the desired frequency range.

\subsection{Final Layout and Results}

In order to simulate the performance of the BPSK modulator we will consider that its input binary wave is periodic (not random). Figure 18 shows the form of such a wave. The power spectral density of this wave is discrete and can be evaluated using Fourier series

$$
f(t)=a_{\mathrm{o}}+\sum_{n=1}^{\infty} a_{n} \cos \left(\frac{2 \pi n t}{T_{\mathrm{o}}}\right)+\sum_{n=1}^{\infty} b_{n} \sin \left(\frac{2 \pi n t}{T_{\mathrm{o}}}\right)
$$

TABLE V Reflection Coefficients at LO and Output Ports of the Active Part of BPSK Modulator.

\begin{tabular}{lrcr}
\hline & \multicolumn{3}{c}{ s-parameters $(\mathrm{dB})$} \\
\cline { 2 - 4 } Frequency $(\mathrm{GHz})$ & \multicolumn{1}{|c}{$\left|s_{44}\right|$} & $\left|s_{55}\right|$ & $\left|s_{33}\right|$ \\
\hline 9.9 & -16.08 & -8.45 & \\
10.0 & -12.45 & -10.11 & -11.62 \\
10.1 & -9.97 & -12.21 & \\
\hline
\end{tabular}




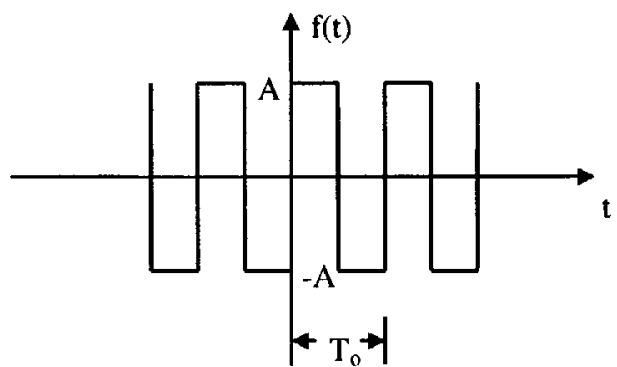

FIGURE 18 Periodic binary input signal.

while it can be found that

$$
\begin{gathered}
a_{\mathrm{o}}=\frac{1}{T_{\mathrm{o}}} \int_{-T_{\mathrm{o}} / 2}^{T_{\mathrm{o}} / 2} f(t) \mathrm{d} t, \quad a_{n}=\frac{2}{T_{\mathrm{o}}} \int_{-T_{\mathrm{o}} / 2}^{T_{\mathrm{o}} / 2} f(t) \cos \left(\frac{2 \pi n t}{T_{\mathrm{o}}}\right) \mathrm{d} t \\
b_{n}=\frac{2}{T_{\mathrm{o}}} \int_{-T_{\mathrm{o}} / 2}^{T_{\mathrm{o}} / 2} f(t) \sin \left(\frac{2 \pi n t}{T_{\mathrm{o}}}\right) \mathrm{d} t
\end{gathered}
$$

Easily can be found that

$$
a_{\mathrm{o}}=a_{n}=0 \text { and } b_{n}=\frac{2 A}{\pi n}\left[1-(-1)^{n}\right]
$$

Figure 19 shows the discrete power spectral density. Therefore the power spectral density of the output signal should look like this. The only difference is that it would be shifted to the LO frequency $\left(f_{\mathrm{LO}}=10 \mathrm{GHz}\right)$.

In Figure 20 the input and output signals can be seen over a period of the binary input. The period of the LO signal is much smaller than the period of the input signal and that is why only the envelope of the BPSK wave can be seen. In Figure 21 a small time slot has been chosen and the change of the phase is obvious. The amplitude of the BPSK wave is not constant because lowpass filters were used so as the bandwidth of the binary input to be reduced.

Figure 22 shows the discrete power spectral density of the periodic binary input signal. Since the bit rate of the binary input signal of the QPSK modulator is $155 \mathrm{Mbits} / \mathrm{sec}$, the

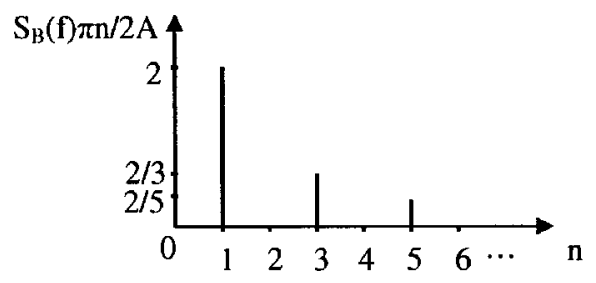

FIGURE 19 Power spectral density of the periodic binary signal. 


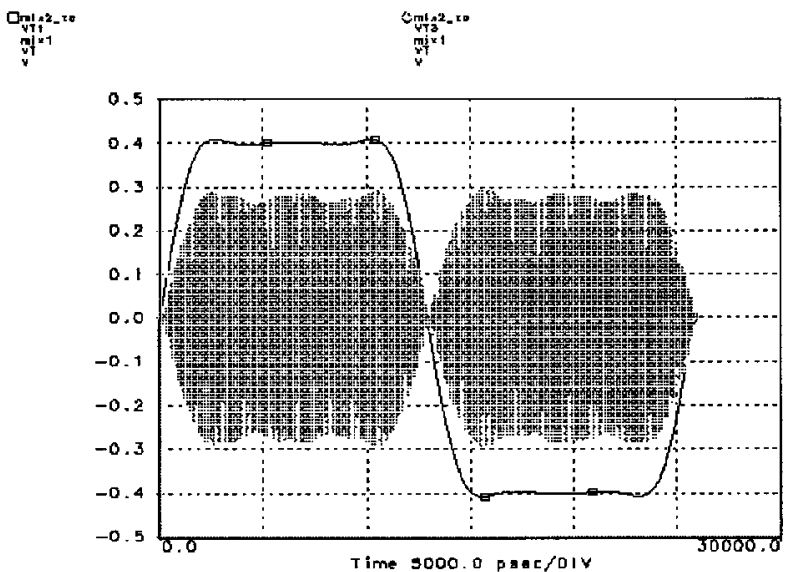

FIGURE 20 Input and output signals during a period of the binary input.

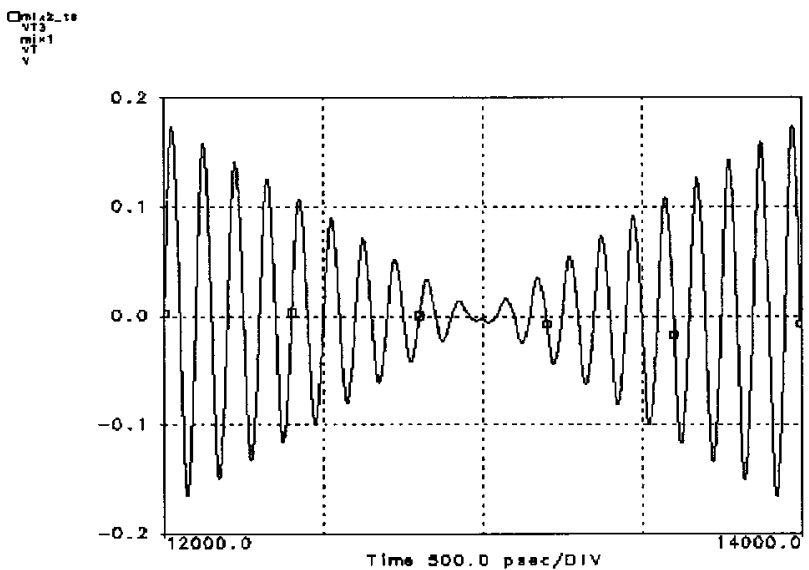

FIGURE 21 BPSK wave during a time slot around the moment that input signal changes.

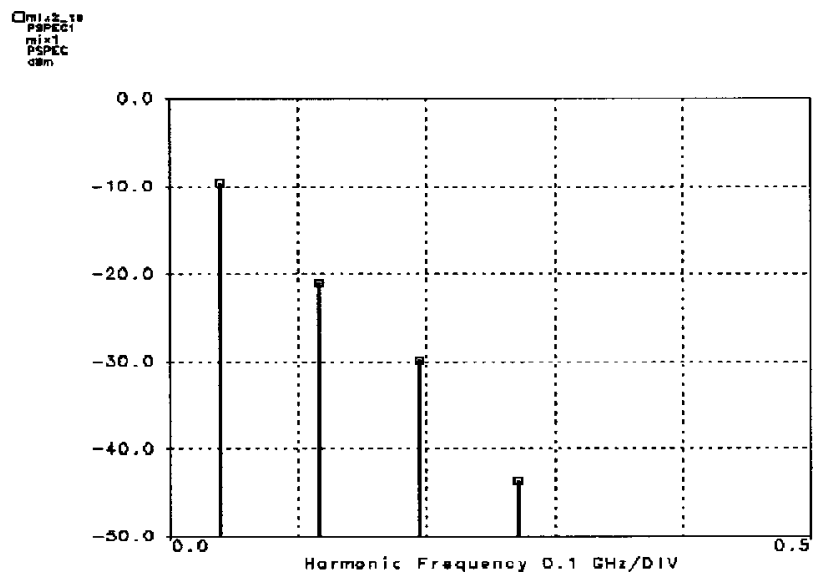

FIGURE 22 Discrete power spectral density of the periodic binary input signal. 


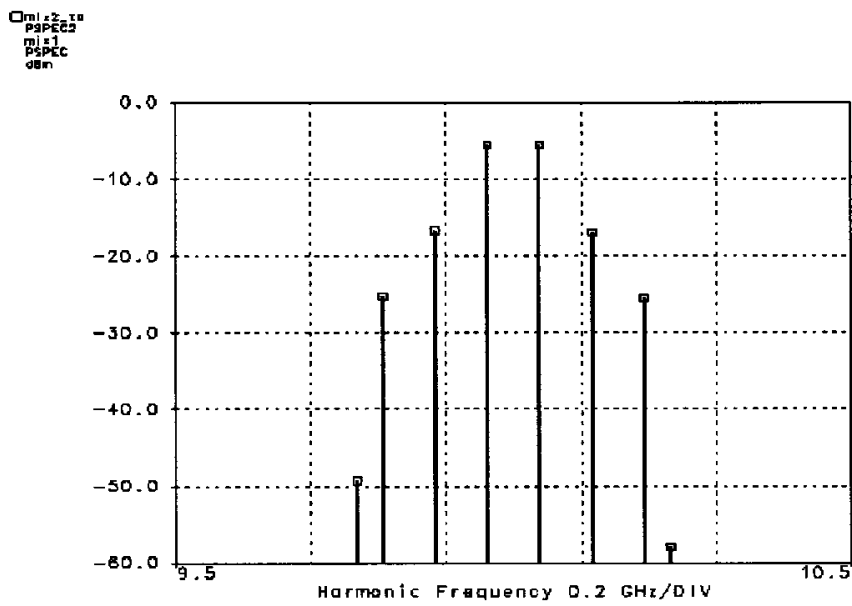

FIGURE 23 Discrete power spectral density of the output BPSK signal.

bit rate of the binary input signals of the BPSK modulators is $77.5 \mathrm{Mbits} / \mathrm{sec}$. Therefore, according to Figures 18 and 19 , it is found that

$$
\frac{T_{\mathrm{o}}}{2}=\frac{1}{77.5} \mu \mathrm{sec}
$$

which means that the first nonzero harmonic of the input signal is at $1 / T_{\mathrm{o}}=38.75 \mathrm{MHz}$, the second at $3 / T_{\mathrm{o}}=116.25 \mathrm{MHz}$, the third at $5 / T_{\mathrm{o}}=193.75 \mathrm{MHz}$ etc.

In Figure 23 the discrete power spectral density of the output signal can be seen. It is similar to the one that was presented in Figure 22, but it is shifted to the LO frequency $\left(f_{\mathrm{LO}}=10 \mathrm{GHz}\right)$. Therefore the first nonzero harmonic of the output signal is at $10 \pm 0.03875 \mathrm{GHz}$, the second at $10 \pm 0.11625 \mathrm{GHz}$, the third at $10 \pm 0.19375 \mathrm{GHz}$ etc.

Figure 24 shows the final layout of the active part of the BPSK modulator. It is placed in such a way that can easily be connected with the other sub-circuits of the design.

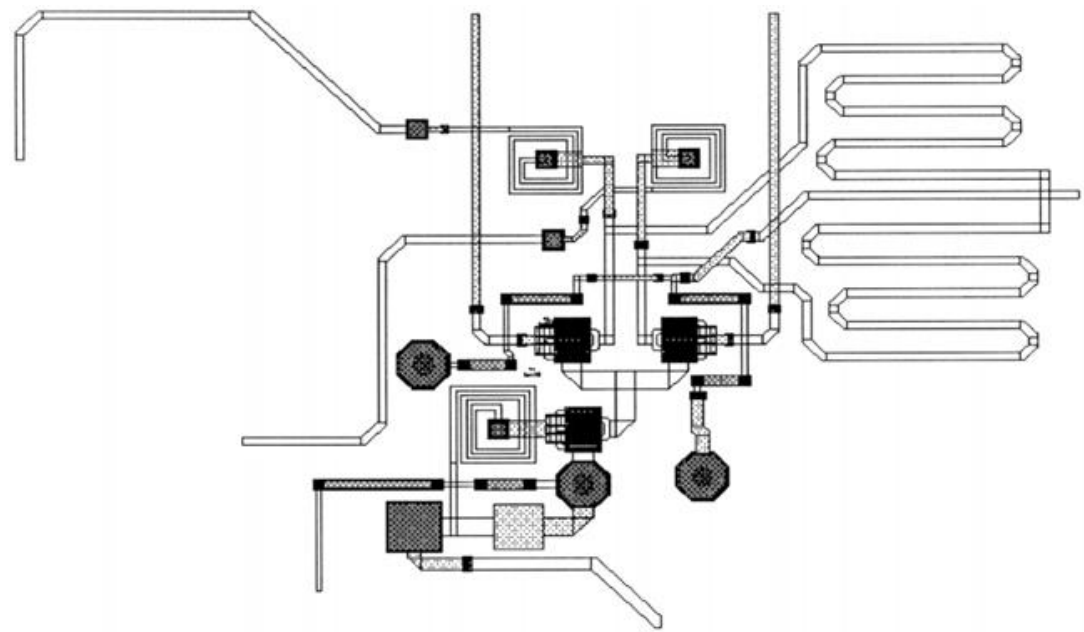

FIGURE 24 Final layout of the active part of the BPSK modulator. 


\section{FINAL LAYOUT AND RESULTS OF THE QPSK MODULATOR}

The final layout of the QPSK-modulator is shown in Figure 25. The sub-circuits (BPSK-modulators, Wilkinson combiner, and 90-deg hybrid) are easily recognized.

The carrier signal, having a frequency of $f_{c}=10 \mathrm{GHz}$, is applied to the pad no. 1, while the output QPSK waveform is extracted from the pad no. 2. The baseband signal $m_{1}(t)$ as well as its reverse $-m_{1}(t)$ are applied to the pads no. 3 and no. 4, respectively. The baseband signal $m_{2}(t)$ as well as its reverse $-m_{2}(t)$ are applied to the pads no. 5 and no. 6 , respectively. The $+5 \mathrm{~V}$ dc voltage is applied to the pads no. 7 and no. 8 , while the $-5 \mathrm{~V}$ dc voltage is applied to the pad no. 9. This integrated circuit measures $3.616 \mathrm{~mm} \times 3.229 \mathrm{~mm}$ and as a result the occupied area is $11.7 \mathrm{~mm}^{2}$.

In Table VI the exact values of the reflection coefficients at the LO input $\left(\left|s_{11}\right|\right)$ and the RF output $\left(\left|s_{22}\right|\right)$ ports are presented. The input impedance at the ports, where the binary signal is applied, is almost $1 \mathrm{k} \Omega$.

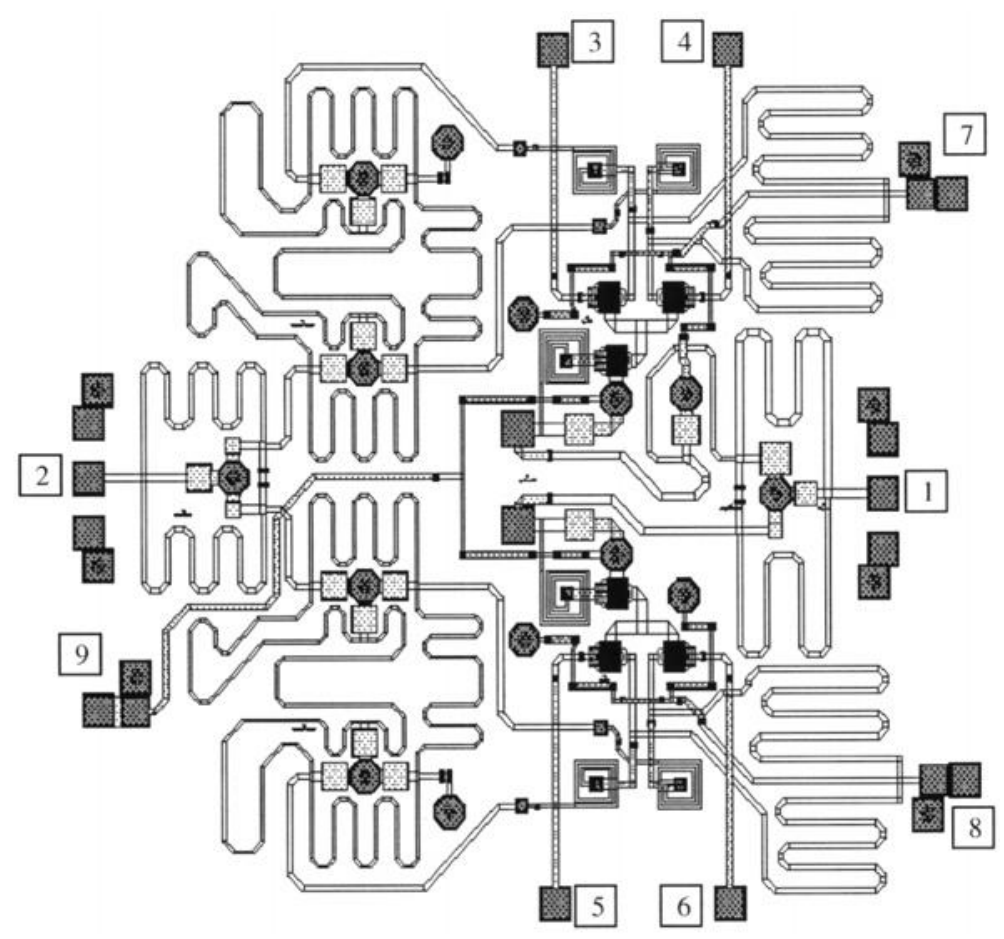

FIGURE 25 Final layout of the QPSK modulator.

TABLE VI Reflection Coefficients at LO and RF Ports of the QPSK Modulator.

\begin{tabular}{lll}
\hline & \multicolumn{2}{c}{ s-parameters $(d B)$} \\
\cline { 2 - 3 } Frequency $(G H z)$ & $\left|s_{22}\right|$ & $\left|s_{11}\right|$ \\
\hline 9.9 & -13.8 & \\
10.0 & -14.6 & -19.0 \\
10.1 & -15.5 & \\
\hline
\end{tabular}




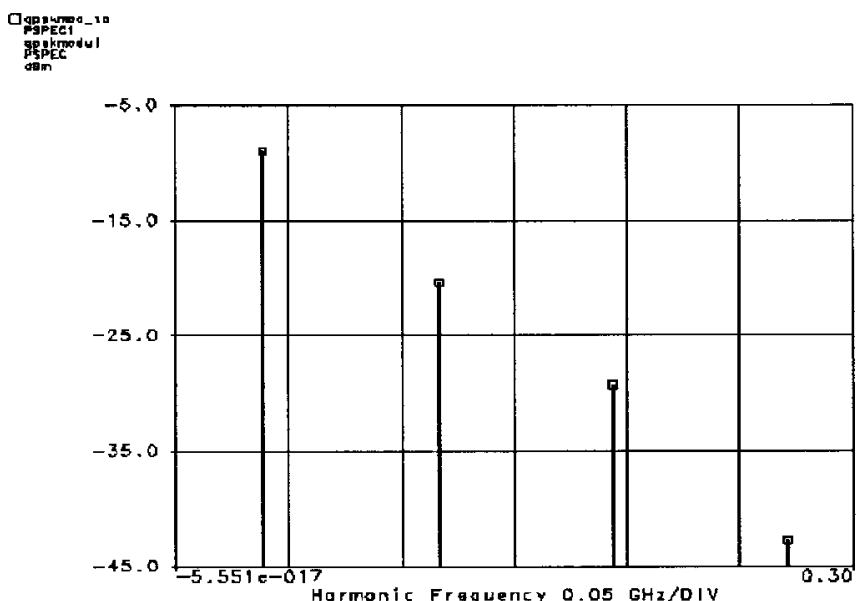

FIGURE 26 Discrete power spectral density of the periodic binary input signal.

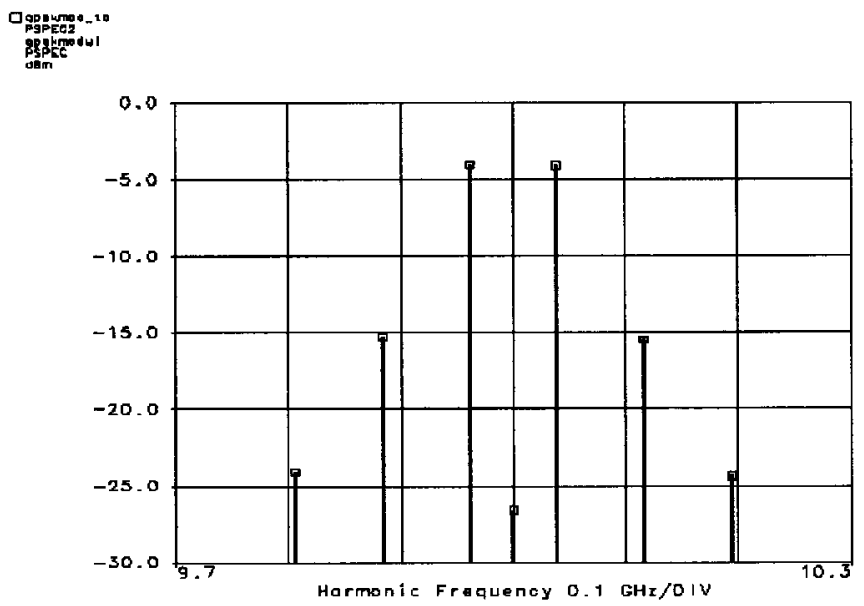

FIGURE 27 Discrete power spectral density of the output QPSK signal.

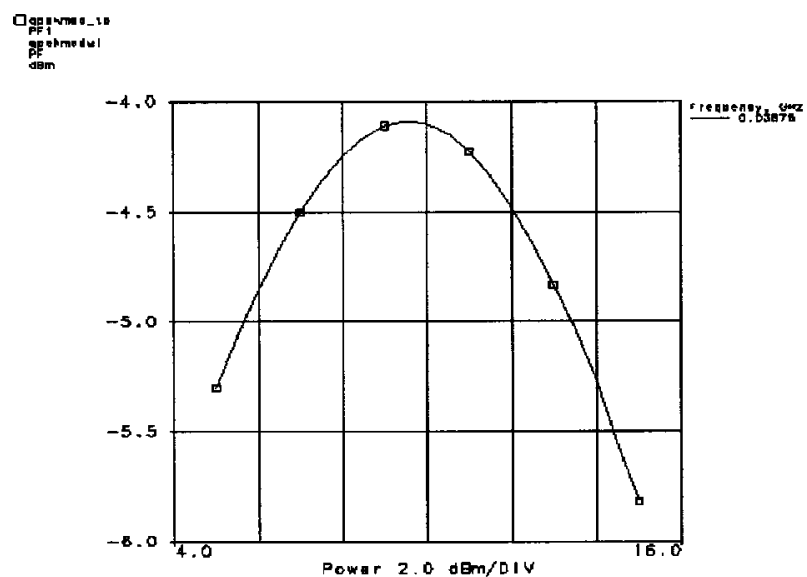

FIGURE 28 Power of the first harmonic of the output signal $(10.03875 \mathrm{GHz}) v s$. the power of the LO signal. 


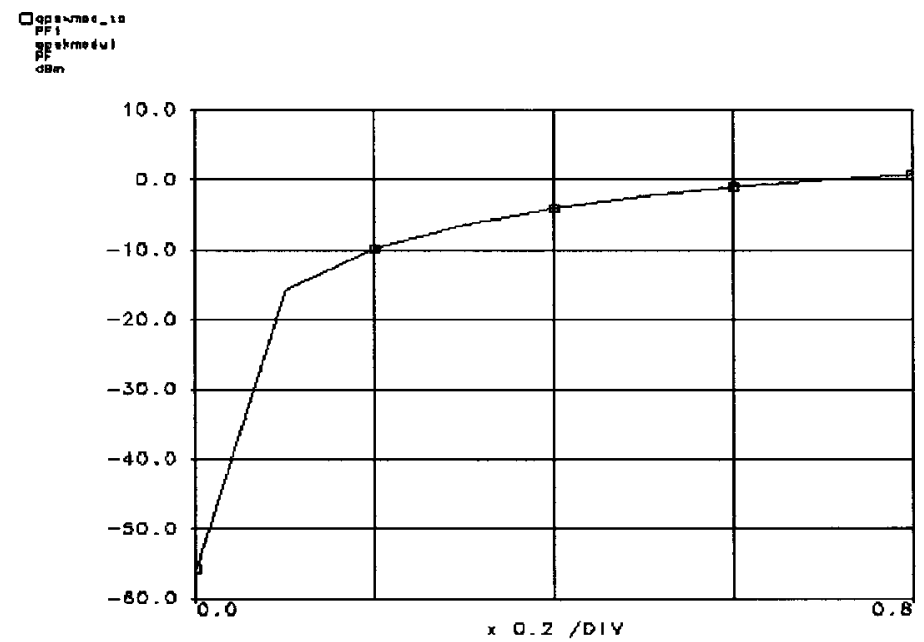

FIGURE 29 Power of the first harmonic of the output signal vs. the amplitude of the input binary wave.

The QPSK modulator was simulated using a periodic input binary wave. Figure 26 shows the discrete power spectral density of the periodic binary input signal (after the demultiplexer).

In Figure 27 the discrete power spectral density of the output signal can be seen. It is similar to the one that was presented in Figure 26, but it is shifted to the LO frequency $\left(f_{\mathrm{LO}}=10 \mathrm{GHz}\right)$. Therefore the first nonzero harmonic of the output signal is at $10 \pm 0.03875 \mathrm{GHz}$, the second at $10 \pm 0.11625 \mathrm{GHz}$, the third at $10 \pm 0.19375 \mathrm{GHz}$ etc.

Figure 28 shows the power of the first harmonic of the output signal $(10.03875 \mathrm{GHz}) v s$. the power of the LO signal and it is obvious that for $P_{\mathrm{LO}} \approx 9 \mathrm{dBm}$ the output power reaches its maximum value.

In Figure 29 again the power of the first harmonic of the output signal $v s$. the amplitude of the input binary wave is presented, while the LO power is considered to be $P_{\mathrm{LO}}=9 \mathrm{dBm}$. It can be seen that when the amplitude of the input binary wave is $0.4 \mathrm{~V}$ the output power is almost $-4 \mathrm{dBm}$. If the amplitude is more than $0.4 \mathrm{~V}$ then the level of the output power is higher, but the DC power consumption is much greater.

Finally, in Table VII the specifications of the QPSK modulator can be seen.

TABLE VII Specifications of the QPSK Modulator.

\begin{tabular}{lll}
\hline Carrier signal (LO) & Frequency & $10 \mathrm{GHz}$ \\
& Power & $9 \mathrm{dBm}$ \\
& Input return loss $\left|s_{11}\right|$ & $-19 \mathrm{~dB}$ \\
Baseband signal (IF) & Bit rate & $155 \mathrm{Mbits} / \mathrm{sec}$ \\
(Before the demultiplexer) & Signal level & $0.4 \mathrm{~V}$ \\
& Input resistance & $1 \mathrm{k} \Omega$ \\
Output signal (RF) & Output power $(10.03875 \mathrm{GHz})$ & $-4.1 \mathrm{dBm}$ \\
& Output return loss $\left|s_{22}\right|$ & $-14.6 \mathrm{~dB}$ \\
& Carrier suppression & $35 \mathrm{~dB}$ \\
& LO harmonics & $<-65 \mathrm{dBm}$ \\
DC signal & Supply Voltage & $+5 \mathrm{~V}$ and $-5 \mathrm{~V}$ \\
& Current consumption & $26 \mathrm{~mA}$ \\
& Power consumption & $130 \mathrm{mWatt}$ \\
\hline
\end{tabular}




\section{CONCLUSION}

A QPSK modulator has been designed using the F-20 process of GEC Marconi. The modulation is performed directly at the RF band ( $\mathrm{X}$ band) and therefore an IF stage is not necessary. The design contains all the necessary sub-circuits except for the demultiplexer and the low-pass filters, which are used so that the bandwidth of the binary waves can be reduced. The circuit needs no external LO coupler and no RF chocks, while the mixers that are contained in the circuit are not the typical Gilbert cells and as a result they occupy a much smaller area (half).

\section{References}

[1] Haykin, S. S. (1983). Communication Systems, John Wiley and Sons.

[2] F-20 Process' manual of GEC Marconi.

[3] Rajesh Mongia, Inder Bahl, Prakash Bhartia (1999). RF and Microwave Coupled-line Circuits, Artech House.

[4] Maas, S. A. (1993). Microwave mixers, 2nd ed., Artech House. 

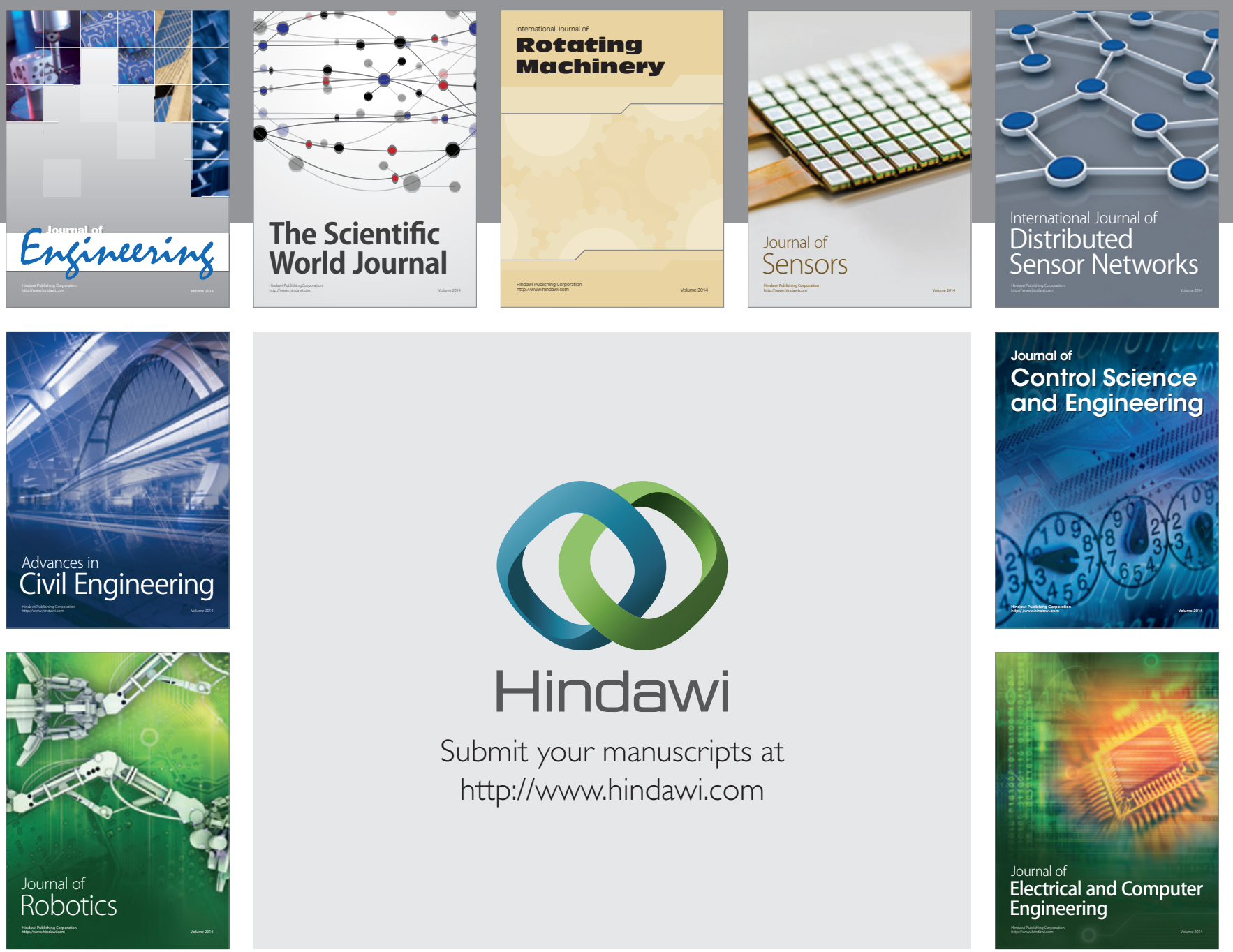

Submit your manuscripts at

http://www.hindawi.com
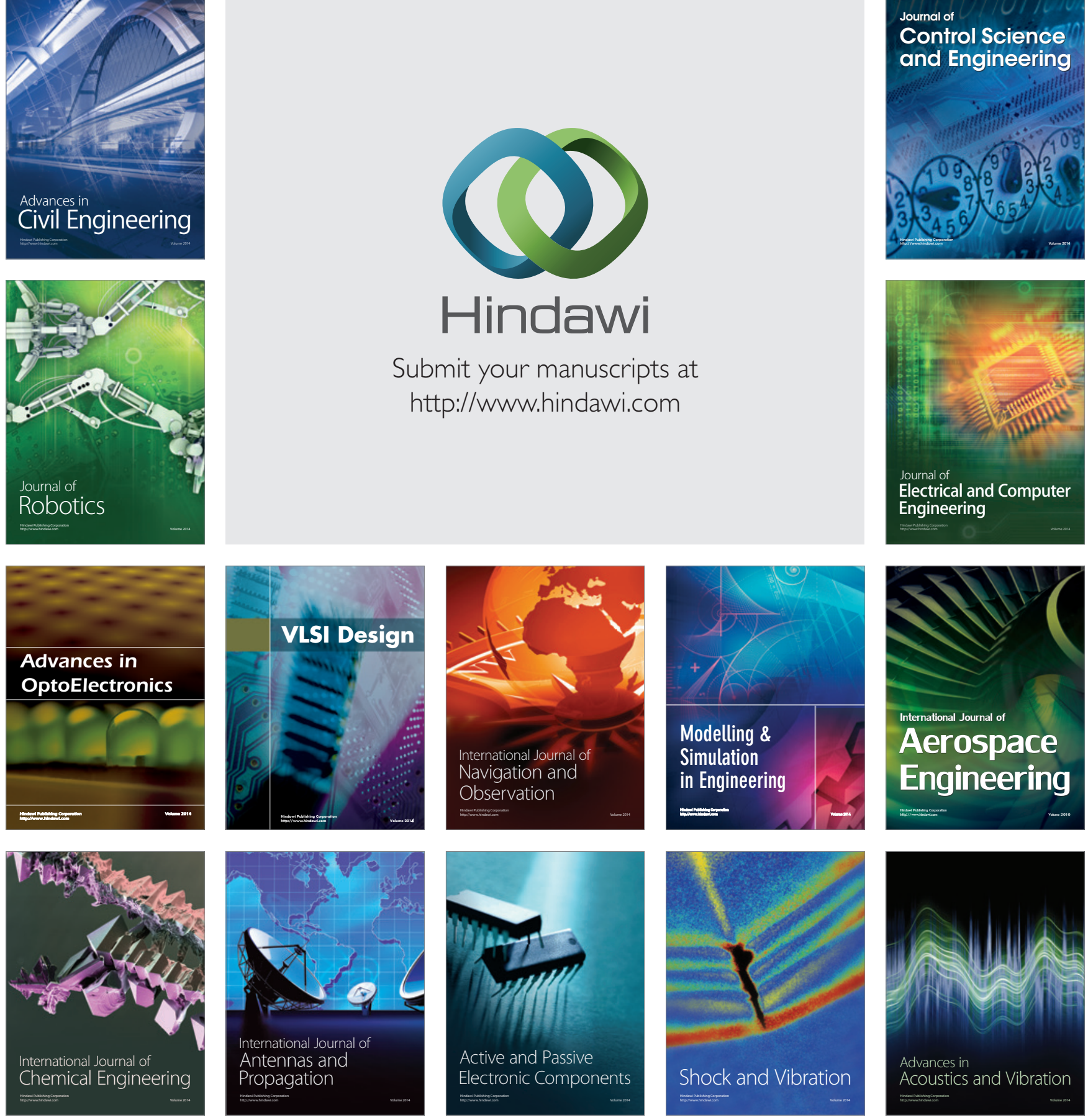